\title{
REGULARITY RESULTS AND LARGE TIME BEHAVIOR FOR INTEGRO-DIFFERENTIAL EQUATIONS WITH COERCIVE HAMILTONIANS.
}

\author{
GUY BARLES, SHIGEAKI KOIKE, OLIVIER LEY, AND ERWIN TOPP
}

\begin{abstract}
In this paper we obtain regularity results for elliptic integrodifferential equations driven by the stronger effect of coercive gradient terms. This feature allows us to construct suitable strict supersolutions from which we conclude Hölder estimates for bounded subsolutions. In many interesting situations, this gives way to a priori estimates for subsolutions. We apply this regularity results to obtain the ergodic asymptotic behavior of the associated evolution problem in the case of superlinear equations. One of the surprising features in our proof is that it avoids the key ingredient which are usually necessary to use the Strong Maximum Principle: linearization based on the Lipschitz regularity of the solution of the ergodic problem. The proof entirely relies on the Hölder regularity.
\end{abstract}

\section{INTRODUCTION.}

In [13, Capuzzo-Dolcetta, Leoni and Porretta prove a surprising regularity result for subsolutions of superquadratic second-order elliptic equations which can be described in the following way. We consider the model equation

$$
\lambda v-\operatorname{Tr}\left(A(x) D^{2} v(x)\right)+b(x)|D v(x)|^{m}=f(x) \quad \text { in } \Omega,
$$

where $\Omega$ is an open subset of $\mathbb{R}^{N}, A, b, f$ are continuous functions in $\Omega, A$ taking values in the set of nonnegative matrices and $b, f$ are real valued, with $b(x) \geq b_{0}>0$ in $\Omega, m>2$ and $\lambda \geq 0$. The function $v: \Omega \rightarrow \mathbb{R}$ is a realvalued solution and $D v, D^{2} v$ denote its gradient and Hessian matrix. In [13], the authors prove that, if $u: \Omega \rightarrow \mathbb{R}$ is a bounded viscosity subsolution of (1.1) then $u$ is locally Hölder continuous with exponent $\alpha:=(m-2)(m-1)^{-1}$ and the local Hölder seminorm depends only on the datum $\left(L^{\infty}\right.$ bounds on $A, f$ and $\left.b_{0}\right)$ but not on any $L^{\infty}$ bound nor oscillation of $u$. Actually this result provides, in many interesting situations, an estimate on the $L^{\infty}$ norm of $u$.

Date: July 8, 2018.

2010 Mathematics Subject Classification. 35R09, 35B51, 35B65, 35D40, 35B10, 35B40.

Key words and phrases. Parabolic Integro-Differential Equations, Regularity, Comparison Principles, Strong Maximum Principles, Large Time Behavior. 
The starting point of the present work was to investigate how such a result could be extended to the case of nonlocal elliptic equations like

$$
\lambda u(x)-I_{x}(u, x)+H(x, D u(x))=0 \quad \text { in } \Omega,
$$

where $\lambda \geq 0$ and $H: \Omega \times \mathbb{R}^{N} \rightarrow \mathbb{R}$ is a continuous nonlinearity having the same properties as $b(x)|p|^{m}-f(x)$ above. The term $I_{x}$ is a nonlocal operator playing the role of the diffusion, defined as follows: for $x, y \in \mathbb{R}^{N}$ and $\phi: \mathbb{R}^{N} \rightarrow \mathbb{R}$ a bounded continuous function which is $C^{2}$ in a neighborhood of $y$, we write

$$
I_{x}(\phi, y)=\int_{\mathbb{R}^{N}}\left[\phi(y+z)-\phi(y)-\mathbf{1}_{B}\langle D \phi(y), z\rangle\right] \nu_{x}(d z),
$$

where $B$ denotes the unit ball and $\left\{\nu_{x}\right\}_{x \in \mathbb{R}^{N}}$ is a family of Lévy measures, see (M1)-(M2) below for precise assumptions. An important example of such nonlocal operator is the case when $\nu_{x}=\nu$ for all $x \in \mathbb{R}^{N}$, with

$$
\nu(d z)=C_{N, \sigma}|z|^{-(N+\sigma)} d z,
$$

where $\sigma \in(0,2)$ and $C_{N, \sigma}$ is a normalizing constant. In that case, for all $x \in \mathbb{R}^{N},-I_{x}=(-\Delta)^{\sigma / 2}$ is the fractional Laplacian of order $\sigma$ (see [22]). By the form of $I_{x}$ in (1.2), we point out that subsolutions of $(\mathrm{P})$ must be defined on $\mathbb{R}^{N}$ or at least in a large enough domain (depending on $\nu_{x}$ ) in order that the nonlocal operator is well-defined.

In [13] and even more in the simplified version given in [4, the authors take advantage of the superquadratic gradient term to construct locally a strict supersolution to (1.1) using power-like functions. The power profile of such supersolutions gives the (local) Hölder regularity for bounded subsolutions of the equation. This proof is based on the leading effect of the gradient term more than on the ellipticity, resembling the behavior of first-order coercive equations (see [3]). The Hölder exponent $(m-2)(m-1)^{-1}$ just comes from a simple balance of powers in (1.1) and this Hölder regularity can be extended up to the boundary of the domain if it is regular enough (see also [4]).

All these arguments seem extendable to the nonlocal framework, and in particular, if we think the nonlocal term as an operator of order $\sigma \in(0,2)$. But here is a key difference which is going to play a double role : first, depending on the support of the measure $\nu_{x}(d z)$, the operator may use values of $u$ outside $\Omega$. This arises, typically, when equation $(\mathrm{P})$ is complemented by an exterior Dirichlet condition (see [8]). Of course, and this is very natural in the case of exterior Dirichlet condition, these outside values cannot be controlled by the equation. Hence, in that case, it is clearly impossible to have results which are independent of the $L^{\infty}$ norm or oscillation of $u$.

On the contrary, this analysis shows that, in principle, this could be possible in the case when the support of the measure is such that the integral of $I_{x}(u, y)$ only takes into account points such that $y+z \in \Omega$, typically when

$$
I_{x}(\phi, y)=C_{N, \sigma} \int_{y+z \in \Omega}\left[\phi(y+z)-\phi(y)-\mathbf{1}_{B}\langle D \phi(y), z\rangle\right]|z|^{-(N+\sigma)} d z
$$


These type of operators are related to "censored processes" in the probabilistic literature : in this context, it means that the jumps processes cannot jump from $\Omega$ to $\Omega^{c}$. We refer to e.g. [11, 23, 24, 25, 30, 29] for more details on such processes. In [11, 25, the censored fractional Laplacian appears in connection with Dirichlet forms; they also appear in the analysis literature as regional Laplacians ([27]) and very naturally in the study of Neumann boundary conditions ([7]). We therefore call censored operators (with respect to $\Omega$ ) the operators which satisfy

$$
x+\operatorname{supp}\left\{\nu_{x}\right\} \subset \Omega, \quad \text { for all } x \in \Omega .
$$

Actually, we remark that we can always reduce to the case of a censored operator by incorporating the integral over the complement of $\Omega$ into the right-hand side $f$ (see Lemma 2.4 below and/or [36]). This "censoring" procedure modifies the right-hand side into a function which blows up at the boundary of $\Omega$ with a rate which is controlled in terms of the singularity of the measure (the $\sigma$ in the fractional Laplacian case) and the oscillation of $u$. Thus, as it can be seen in [13, the presence of these unbounded ingredients in the equation restricts the expected values of the Hölder exponent if we wish a result which holds up to the boundary. Moreover, the same effect arises even for nonlocal operators which are originally censored, since the proof of the Hölder regularity consists in localizing, typically in some ball included in $\Omega$ and, at this step also, the values of $u$ outside the ball creates essentially the same difficulty as the one described above : if we want to write the nonlocal equation as a censored equation in the ball, then this mechanically changes the "natural" Hölder exponent because of the righthand side which blows up at the boundary of the ball.

All these difficulties explain all the different formulations we give for some results but also the nature of the Hölder exponent we obtain. To be more specific, we consider the basic model equation

$$
\lambda u(x)+a(x)(-\Delta)^{\sigma / 2} u(x)+b(x)|D u(x)|^{m}=f(x) \quad \text { in } \Omega,
$$

where $\lambda, b, f$ are as in (1.1) and $a$ is a continuous real-valued function with $a \geq 0$ in $\Omega$. The role of the superquadraticity in (1.1) is played by a superfractional growth condition on the gradient, which is encoded by $m$ in (1.4) through the assumption

$$
m>\sigma
$$

and the strict positivity requirement on $b$. The difficulties we mention above on the nonlocality have a price and this price is a "less natural" Hölder exponent $(m-\sigma) / m$ for subsolutions to (1.4). Nevertheless, we can get interior Hölder regularity results with "more natural" exponents $(m-\sigma) /(m-1)$ if $\sigma>1$, Lipschitz continuity if $\sigma<1$, and any exponent in $(0,1)$ for $\sigma=1$, since localization arguments are unnecessary in this situation. Finally, we point out that in the case of censored operators (here if $(-\Delta)^{\sigma / 2}$ is replaced 
by the operator given by (1.3)), we recover a complete control on the oscillation of $u$ on $\bar{\Omega}$ as a consequence of the form of the estimates (see Corollary 2.9 below).

It is worth pointing out that our results share (with some limitations we described above) the same interesting consequence as the ones of [13], namely a control on the oscillation of (sub)solutions to (1.1) inside $\Omega$ (i.e. at least locally) which is stable as $\lambda \rightarrow 0^{+}$. This feature has important applications on the study of large time behavior for associated parabolic problems and homogenization because of the importance of the ergodic problem.

We are able to provide global oscillation bounds satisfying this stability property for some class of problems $(\mathbb{P})$ as, for example, equations associated to censored operators and obviously for equations set in the whole space $\mathbb{R}^{N}$. This contrasts with the results obtained by Cardaliaguet and Rainer [15] (see also [14]), where the authors obtain very interesting regularity results for (parabolic) superquadratic integro-differential equations using a probabilistic approach, but where their Hölder estimates depend on the $L^{\infty}$ norm of the solution.

In the second part of this paper, we present an application of our regularity results to the study of the large time behavior for Cauchy problems

$$
\partial_{t} u(x, t)-I_{x}(u(\cdot, t), x)+H(x, D u(x, t))=0 \quad \text { in } Q,
$$

where $Q=\mathbb{R}^{N} \times(0,+\infty)$. The asymptotic behavior of the nonlocal evolution problem is also motivated by its second-order parallel, as the model equation

$$
\partial_{t} u(x, t)-\operatorname{Tr}\left(A(x) D^{2} u(x, t)\right)+b(x)|D u(x, t)|^{m}=f(x) \text { in } Q .
$$

In the superquadratic case $m>2$, this evolution equation is also influenced by the stronger effect of the first-order term. This can be seen in the paper of Barles and Souganidis [10, where the authors study general equations including (1.1) and (1.6), obtain Lipschitz bounds for the solutions and prove that, in the periodic setting, the solution approaches to the solution of the so-called ergodic problem as $t \rightarrow+\infty$. This ergodic problem is solved by passing to the limit as $\lambda \rightarrow 0^{+}$in equation (1.1), which is possible by the compactness given by the Lipschitz bounds which are independent of $\lambda$. A second key ingredient in the analysis of the ergodic problem and the large time behavior of (1.6) is the Strong Maximum Principle ([2]).

Similar methods and results to [10] are obtained in [35] in the context of Cauchy-Dirichlet second-order evolution problems in bounded domains. In the nonlocal context, analogous ergodic large time behavior for evolution problems are available. For instance, in [6] the authors follow the arguments of [10, using the Lipschitz regularity results given in 5, which allows to "linearize" the equation in order to apply the Strong Maximum Principle of [17.

In this paper we also follow the lines of [10] to prove the ergodic asymptotic behavior. However, contrarily to 10 or [6], we do not use the Strong Maximum Principle in the same way : we do not perform any "linearization" 
of the equation (which would have required Lipschitz bounds) and therefore we are able to provide results which just use the Hölder regularity of the solutions. This proof requires slightly stronger assumptions on the nonlocal operator since we have to be able to use the Strong Maximum Principle à la Coville [18, 19] and to do so, we need the support of the measure defining the nonlocal operator to satisfy an "iterative covering property". Though a restriction, this property allows us to study the large time behavior for equations associated to very degenerate $x$-dependent nonlocal operators and $x$-dependent Hamiltonians with a higher degree of coercivity.

Of course, comparison principles are of main importance in this method and for this reason we should focus on a particular class of $x$-dependent nonlocal operators in Lévy-Ito form (see (2.43)). We refer to [9] for comparison results associated to these operators.

The paper is organized as follows: Section 2 is entirely devoted to the regularity results for the stationary problem. In section 3 we provide the comparison principle and well-possedness of the evolution problem. Finally, the large time behavior for this problem is presented in section 4, where the mentioned version of the strong maximum principle is established.

Basic Notation. For $x \in \mathbb{R}^{N}$ and $r>0$, we denote $B_{r}(x)$ as the open ball centered at $x$ with radius $r$. We just write $B_{r}$ for $B_{r}(0)$ and $B$ for $B_{1}(0)$.

Let $\Omega \subset \mathbb{R}^{N}$. We denote as $d_{\Omega}$ the signed distance function to $\partial \Omega$ which is nonnegative in $\bar{\Omega}$. For $\delta>0$, we also denote $\Omega^{\delta}=\left\{x \in \Omega: d_{\Omega}(x)>\delta\right\}$. For any $u: \Omega \rightarrow \mathbb{R}$, the oscillation of $u$ over $\Omega$ is defined by

$$
\operatorname{osc}_{\Omega} u=\sup _{\Omega} u-\inf _{\Omega} u \text {. }
$$

For $x, \xi, p \in \mathbb{R}^{N}, A \subset \mathbb{R}^{N}$ and $\phi$ a bounded function, we define

$$
I_{\xi}[A](\phi, x, p)=\int_{\mathbb{R}^{N} \cap A}\left[\phi(x+z)-\phi(x)-\mathbf{1}_{B}\langle p, z\rangle\right] \nu_{\xi}(d z) .
$$

We write in a simpler way $I_{\xi}[A](\phi, x)=I_{\xi}[A](\phi, x, D \phi(x))$ when $\phi \in$ $L^{\infty}\left(\mathbb{R}^{N}\right) \cap C^{2}\left(B_{\delta}\right)$ for some $\delta>0, I_{\xi}(\phi, x, p)=I_{\xi}\left[\mathbb{R}^{N}\right](\phi, x, p)$ when $A=\mathbb{R}^{N}$ and $I=I_{\xi}$ if $\nu_{\xi}=\nu$ does not depend on $\xi$. Note that with these notations, $I_{x}(\phi, x)=I_{x}\left[\mathbb{R}^{N}\right](\phi, x, D \phi(x))$ for $\phi$ bounded and smooth at $x$ (see (1.2) $)$.

This paper is based on the viscosity theory to get the results. We refer to [20, 3, 31] for the definition and main results of the classical theory, and to $[9,8,1,33$, 34] for the nonlocal setting. Following the definition introduced in the mentioned references, we always assume a viscosity subsolution is upper semicontinuous and a viscosity supersolution is lower semicontinuous in the set where the equation takes place.

\section{Regularity.}

2.1. Assumptions and Main Regularity Results. Let $\sigma \in(0,2)$ fixed. Recalling $I_{x}$ defined in (1.2), we assume the following conditions over the family $\left\{\nu_{x}\right\}_{x}$ 
(M1) For all $R>0$ and $\alpha \in[0,2]$, there exists a constant $C_{R}>0$ such that, for all $\delta>0$ we have

$$
\sup _{x \in \bar{B}_{R}} \int_{B_{\delta}^{c}} \min \left\{1,|z|^{\alpha}\right\} \nu_{x}(d z) \leq C_{R} h_{\alpha, \sigma}(\delta),
$$

where $h_{\alpha, \sigma}(\delta)$ is defined for $\delta>0$ as

$$
h_{\alpha, \sigma}(\delta)=\left\{\begin{array}{cc}
\delta^{\alpha-\sigma} & \text { if } \alpha<\sigma \\
|\ln (\delta)|+1 & \text { if } \alpha=\sigma \\
1 & \text { if } \alpha>\sigma
\end{array}\right.
$$

and where we use the convention $|z|^{\alpha}=1, z \in \mathbb{R}^{N}$ when $\alpha=0$.

(M2) For all $R>0$ and $\alpha \in(\sigma, 2]$ there exists a constant $C_{R}>0$ such that, for all $\delta \in(0,1)$ we have

$$
\sup _{x \in \bar{B}_{R}} \int_{B_{\delta}}|z|^{\alpha} \nu_{x}(d z) \leq C_{R} \delta^{\alpha-\sigma}
$$

Assumptions (M1) and (M2) say the nonlocal operator $I_{x}$ is at most of order $\sigma$, locally in $x \in \mathbb{R}^{N}$. Concerning this last fact, we remark that in the case $\nu_{x}$ is symmetric and $\sigma \in(0,1), I_{x}$ defined in (1.2) can be written as

$$
I_{x}(\phi, y)=\int_{\mathbb{R}^{N}}[\phi(y+z)-\phi(y)] \nu_{x}(d z)
$$

for all $y \in \mathbb{R}^{N}$ and $\phi$ bounded and $C^{1}$ in a neighborhood of $y$. Since our interest is to keep $I_{x}$ as a nonlocal operator of order $\sigma$, we adopt this formula as a definition for $I_{x}$ in the case $\sigma \in(0,1)$, even if $\nu_{x}$ is not symmetric.

In order to expand the application of our results, we consider an open set $\Omega \subseteq \mathbb{R}^{N}$ not necessarily bounded, and $H$ satisfying the growth condition

$$
H(x, p) \geq b_{0}|p|^{m}-A\left(d_{\Omega}(x)^{-\theta}+1\right), \quad \text { for } x \in \Omega, p \in \mathbb{R}^{N},
$$

where $b_{0}, A>0$ and $0 \leq \theta<m$.

We first concentrate in regularity results in the superlinear case

$$
m>\max \{1, \sigma\},
$$

which encodes the coercivity of the Hamiltonian, see Theorems 2.1 and 2.2 below. Note that, in Section 2.5, we state also a result in the sublinear case and, in Section 2.6, we extend our results in the superlinear case to Lévy-Ito operators.

Over the exponent $\theta$, we assume $0 \leq \theta<m$ in order to state the blow-up behavior at the boundary of the right-hand side. Thus, our arguments rely over the (more general) equation

$$
-I_{x}(u, x)+b_{0}|D u(x)|^{m}=A\left(d_{\Omega}(x)^{-\theta}+1\right), \quad x \in \Omega .
$$


In principle, due to the nonlocal nature of $I_{x}$, the function $u$ satisfying the above equation should be defined not only in $\Omega$ but on the set

$$
\Omega_{\nu}=\Omega \cup \bigcup_{x \in \bar{\Omega}}\left\{x+\operatorname{supp}\left\{\nu_{x}\right\}\right\}
$$

which, loosely speaking, represents the reachable set from $\Omega$ through $\nu$.

The following result states the regularity up to the boundary for subsolutions of problem (P').

Theorem 2.1. Let $\Omega \subseteq \mathbb{R}^{N}$ be a bounded domain, $A, b_{0}>0$ and $\sigma \in(0,2)$. Let $\left\{\nu_{x}\right\}_{x \in \mathbb{R}^{N}}$ be a family of measures satisfying (M1)-(M2) relative to $\sigma$, and $I_{x}$ defined as in (1.2) if $\sigma \geq 1$ and as (2.2) if $\sigma<1$, associated to $\left\{\nu_{x}\right\}_{x \in \mathbb{R}^{N}}$. Let $m>\max \{1, \sigma\}, \theta \in[0, m)$, and define

$$
\gamma_{0}=\min \{(m-\sigma) / m,(m-\theta) / m\} .
$$

Then, any bounded viscosity subsolution $u: \mathbb{R}^{N} \rightarrow \mathbb{R}$ to the problem ( $\left(\mathrm{P}^{\prime}\right)$ is locally Hölder continuous in $\Omega$ with Hölder exponent $\gamma_{0}$ as in (2.5), and Hölder seminorm depending on $\Omega$, the data and $\operatorname{osc}_{\Omega_{\nu}}(u)$, with $\Omega_{\nu}$ defined as in (2.4).

Moreover, if $\Omega$ has a $C^{1,1}$ boundary, then $u$ can be extended to $\bar{\Omega}$ as a Hölder continuous function of exponent $\gamma_{0}$.

A second result states interior Hölder regularity for subsolutions of ( $\left(\mathrm{P}^{\prime}\right)$ with a Hölder exponent which is more natural to the balance between the order of the nonlocal operator and the Hamiltonian.

Theorem 2.2. Let $\Omega \subseteq \mathbb{R}^{N}$ be a bounded domain, $A, b_{0}>0$ and $\sigma \in(0,2)$. Let $\left\{\nu_{x}\right\}_{x \in \mathbb{R}^{N}}$ be a family of measures satisfying (M1)-(M2) relative to $\sigma$, and $I_{x}$ defined as in (1.2) if $\sigma \geq 1$ and as (2.2) if $\sigma<1$, associated to $\left\{\nu_{x}\right\}_{x \in \mathbb{R}^{N}}$. Let $m>\max \{1, \sigma\}$ and $\theta \in[0, m)$. Define

$$
\tilde{\gamma}_{0}=\tilde{\gamma}_{0}(\sigma, m)=\left\{\begin{array}{cl}
(m-\sigma) /(m-1) & \text { if } \sigma>1 \\
\in(0,1) & \text { if } \sigma=1 \\
1 & \text { if } \sigma<1
\end{array}\right.
$$

and consider

$$
\gamma_{0}=\min \left\{\tilde{\gamma}_{0},(m-\theta) / m\right\} .
$$

Then, any bounded viscosity subsolution $u: \mathbb{R}^{N} \rightarrow \mathbb{R}$ to the equation ( $(\mathrm{P})$ is locally Hölder continuous in $\Omega$ with exponent $\gamma_{0}$ given by (2.7), and Hölder seminorm depending on the data, $\Omega$ and $\operatorname{osc}_{\Omega_{\nu}}(u)$, where $\Omega_{\nu}$ is defined as in (2.4).

Note that for the same data, $\gamma_{0}$ defined in (2.7) is always bigger or equal than $\gamma_{0}$ defined in (2.5), and therefore, the interior Hölder exponent given by Theorem 2.2 is better than the one given by Theorem 2.1 .

Remark 2.3. Theorems 2.1 and 2.2 can be extended to unbounded domains. In fact, if $\Omega$ is unbounded, arguing over a bounded set $\Omega^{\prime} \subset \Omega$ we can apply the method used in the above theorems to conclude the corresponding local 
Hölder regularity results for $\Omega$. Moreover, if $\partial \Omega$ has uniform $C^{1,1}$ bounds, and if (M1)-(M2) hold with $C_{R}$ independent of $R$, then we have global Hölder estimates for bounded subsolutions to $\left(\mathrm{P}^{\prime}\right)$, in the flavour of Theorem [2.1.

Since our aim is to include in our regularity results nonlocal operators of censored nature, we provide here a more accurate definition of such an operator. Recalling definition (2.4), we say that $I_{x}$ is of censored nature relative to $\Omega$ if the family $\left\{\nu_{x}\right\}_{x \in \mathbb{R}^{N}}$ defining $I_{x}$ satisfies the condition

$$
\Omega_{\nu}=\Omega \text {. }
$$

The idea is to set up the problem to provide an unified proof of Theorem 2.1 for censored and noncensored operators. This is possible after a "censoring" procedure we explain now. Let $\left\{\nu_{x}\right\}_{x \in \mathbb{R}^{N}}$ a family of Lévy measures and $\Omega \subseteq \mathbb{R}^{N}$ an open set. For each $\xi \in \mathbb{R}^{N}$ we define the censored measure respect to $\Omega$ and $\xi$ as

$$
\tilde{\nu}_{\xi}(d z)=\mathbf{1}_{\Omega-\xi}(z) \nu_{\xi}(d z) .
$$

For $\xi, x \in \mathbb{R}^{N}, \delta>0$ and a bounded function $\phi \in C^{2}\left(\bar{B}_{\delta}(x)\right)$, we define

$$
\begin{aligned}
\tilde{I}_{\xi}(\phi, x) & =\int_{\mathbb{R}^{N}}\left[\phi(x+z)-\phi(x)-\mathbf{1}_{B}\langle D \phi(x), z\rangle\right] \tilde{\nu}_{\xi}(d z) \\
& =\int_{\Omega-\xi}\left[\phi(x+z)-\phi(x)-\mathbf{1}_{B}\langle D \phi(x), z\rangle\right] \nu_{\xi}(d z) .
\end{aligned}
$$

Of special interest is the censored operator $I_{\Omega}$ defined as

$$
I_{\Omega}(\phi, x)=\tilde{I}_{x}(\phi, x), \quad x \in \bar{\Omega},
$$

from whose definition we note that $I_{\Omega}(\phi, x)=I_{x}[\Omega-x](\phi, x)$.

Note that if $\left\{\nu_{x}\right\}_{x \in \mathbb{R}^{N}}$ satisfies (M1) and (M2), then $\left\{\tilde{\nu}_{x}\right\}_{x \in \mathbb{R}^{N}}$ satisfies (M1) and (M2) with the same constants $C_{R}$. Thus, the next lemma allows us to reduce general nonlocal equations like $\left(\overline{\mathrm{P}^{\prime}}\right)$ to the censored case.

Lemma 2.4. (Censoring the Equation) Let $\Omega \subset \mathbb{R}^{N}$ open and bounded, $\sigma \in(0,2)$ and $\left\{\nu_{x}\right\}_{x \in \mathbb{R}^{N}}$ a family of measures satisfying (M1)-(M2) related to $\sigma$. Let $I_{x}$ be as in (1.2), (2.2) associated to $\left\{\nu_{x}\right\}_{x \in \mathbb{R}^{N}}$. Let $m>\sigma, \beta_{0}>0$ and for $f: \Omega \rightarrow \mathbb{R}$ locally bounded, let $u: \mathbb{R}^{N} \rightarrow \mathbb{R}$ be a bounded viscosity subsolution to

$$
-I_{x}(u, x)+\beta_{0}|D u(x)|^{m}=f(x), \quad x \in \Omega .
$$

Then, there exists $C>0$ (depending on $\Omega$ and $\beta_{0}$ ) such that the function $u$ restricted to $\Omega$ satisfies, in the viscosity sense, the inequality

$$
-I_{\Omega}(u, x)+\frac{\beta_{0}}{2}|D u(x)|^{m} \leq f(x)+C\left(\operatorname{osc}_{\Omega_{\nu}}(u)+1\right) d_{\Omega}(x)^{-\sigma}, \quad x \in \Omega,
$$

where $I_{\Omega}$ is defined in (2.11) and $\Omega_{\nu}$ is defined in (2.4).

Proof: For simplicity, we present the proof for classical subsolutions. The rigorous proof follows easily by using classical viscosity techniques (for instance, see [36]). We also focus on the case $\sigma \geq 1$. 
Using (2.12), for each $x \in \Omega$ we have

$$
\begin{aligned}
& -I_{\Omega}(u, x)+\beta_{0}|D u(x)|^{m} \\
\leq & f(x)+\int_{\Omega^{c}-x}(u(x+z)-u(x)) \nu_{x}(d z)+|D u(x)| \int_{B \cap\left(\Omega^{c}-x\right)}|z| \nu_{x}(d z) \\
\leq & f(x)+C\left(\operatorname{osc}_{\Omega_{\nu}}(u) d_{\Omega}(x)^{-\sigma}+|D u(x)| h_{1, \sigma}\left(d_{\Omega}(x)\right)\right),
\end{aligned}
$$

where $C>0$ comes from the application of (M1) and depends only on $\Omega$. Now, by Young's inequality, there exists $C\left(\beta_{0}\right)$ such that

$$
|D u(x)| d_{\Omega}(x)^{1-\sigma} \leq \frac{\beta_{0}}{2}|D u(x)|^{m}+C\left(\beta_{0}\right) h_{1, \sigma}\left(d_{\Omega}(x)\right)^{m /(m-1)} .
$$

At this point, we note that since $m>\sigma$ we have $m(1-\sigma) /(m-1) \geq-\sigma$. Then, if $\sigma>1$, using (2.1) we can write

$$
h_{1, \sigma}\left(d_{\Omega}(x)\right)^{m /(m-1)}=d_{\Omega}(x)^{m(1-\sigma) /(m-1)} \leq d_{\Omega}(x)^{-\sigma},
$$

meanwhile if $\sigma=1$, we get

$$
h_{1, \sigma}\left(d_{\Omega}(x)\right)^{m /(m-1)}=\left(\left|\log \left(d_{\Omega}(x)\right)\right|+1\right)^{m /(m-1)} \leq C d_{\Omega}(x)^{-\sigma},
$$

where $C>0$ depends only on $m$. Thus, using these estimates we conclude the result for the case $\sigma \geq 1$.

The case $\sigma<1$ follows the same ideas but with easier computations because of the first order finite difference of the integrand defining $I_{x}$, see (2.2).

2.2. Key Technical Lemmas. We start with some notation: for $r>0$ and $x_{0} \in \mathbb{R}^{N}$, define

$$
d_{0}(x)=\left|x-x_{0}\right| \quad \text { and } \quad d_{r}(x)=r-d_{0}(x),
$$

that is, for $x \in B_{r}\left(x_{0}\right), d_{0}(x)$ represents the distance of $x$ to the center of the ball, meanwhile $d_{r}(x)=d_{B_{r}\left(x_{0}\right)}(x)$ is the distance of $x$ to the boundary of the ball. We define $w$ as

$$
w=w_{1}+w_{2}
$$

where, for $C_{1}, \gamma>0$ and $C_{2} \geq 0$ we consider

$$
\begin{aligned}
& w_{1}(x)= \begin{cases}C_{1} d_{0}(x)^{\gamma} & x \in \bar{B}_{r}\left(x_{0}\right) \\
C_{1} r^{\gamma} & x \in \bar{B}_{r}^{c}\left(x_{0}\right)\end{cases} \\
& w_{2}(x)= \begin{cases}C_{1}\left(r^{\gamma}-d_{r}(x)^{\gamma}\right) & x \in \bar{B}_{r}\left(x_{0}\right) \\
C_{1} r^{\gamma}+C_{2} & x \in \bar{B}_{r}^{c}\left(x_{0}\right) .\end{cases}
\end{aligned}
$$

We note that $w_{1}$ and $w_{2}$ (when $C_{2}=0$ ) are Hölder continuous in $\mathbb{R}^{N}$ with exponent $\gamma$. If $C_{2}>0, w_{2}$ is $\gamma$-Hölder in $B_{r}\left(x_{0}\right)$ and it has a discontinuity on $\partial B_{r}\left(x_{0}\right)$. In any case, both $w_{1}$ and $w_{2}$ (for any $C_{2} \geq 0$ ) are smooth in $B_{r}\left(x_{0}\right) \backslash\{0\}$.

For $x \in B_{r}\left(x_{0}\right)$ consider $\varrho$ defined as

$$
\varrho(x)=\frac{1}{4} \min \left\{d_{0}(x), d_{r}(x)\right\}
$$


Of course, $w$ depends on the particular choice of $\gamma, r, x_{0}, C_{1}, C_{2}$, meanwhile $\varrho$ depends on $r$ and $x_{0}$, but we omit these dependences for simplicity of the notation.

We remark that if $\left|x-x_{0}\right| \leq r / 2$ then $\varrho(x)=d_{0}(x) / 4$, meanwhile if $\left|x-x_{0}\right|>r / 2$ we have $\varrho(x)=d_{r}(x) / 4$.

The goal is to prove that $w$ is a supersolution of $\left(\overline{\mathrm{P}^{\prime}}\right)$. The following key lemma gives us a first useful estimate for the nonlocal term applied to $w$.

Lemma 2.5. Let $\sigma \in(0,2)$ and a family of measures $\left\{\nu_{x}\right\}_{x \in \mathbb{R}^{N}}$ satisfying (M1), (M2) relative to $\sigma$. Let $I_{x}$ as in (1.2), (2.2) associated to $\left\{\nu_{x}\right\}_{x \in \mathbb{R}^{N}}$. Let $x_{0} \in \mathbb{R}^{N}, r \in(0,1), \gamma \in(0,1], C_{1}>0, C_{2} \geq 0$, and consider $w$ as in (2.14) and $\varrho$ as in (2.16) associated to these parameters. Then, there exists a constant $C>0$ (not depending on $r, C_{1}$ and $C_{2}$ ) such that

$$
\sup _{\xi \in B_{1}(x)}\left\{I_{\xi}(w, x)\right\} \leq C \begin{cases}C_{1} \varrho^{\gamma-1}(x) h_{1, \sigma}(\varrho(x)) & \text { if } C_{2}=0, \sigma \geq 1 \\ C_{1} h_{\gamma, \sigma}(\varrho(x)) & \text { if } C_{2}=0, \sigma<1 \\ \left(C_{1}+C_{2}\right) \varrho(x)^{-\sigma} & \text { if } C_{2}>0\end{cases}
$$

for each $x \in B_{r}\left(x_{0}\right) \backslash\left\{x_{0}\right\}$.

Proof: Denote $R=\left|x_{0}\right|+1$. We remark that $C_{R}$ in the arguments to come is a generic constant depending on $R$ through the constants arising in (M1) and (M2). The constant $C$ arising in the proof is a positive constant independent of $x, R, r, C_{1}$ or $C_{2}$.

Consider $x \in B_{r}\left(x_{0}\right) \backslash\left\{x_{0}\right\}$. For each $\xi \in B_{1}(x)$, by definition of $w$ we can write

$$
I_{\xi}(w, x)=I_{\xi}\left(w_{1}, x\right)+I_{\xi}\left(w_{2}, x\right),
$$

where $w_{i}, i=1,2$ are defined in (2.15). In what follows, we are going to estimate the integrals in the right-hand side of the above expression.

1.- Estimate for $I_{\xi}\left(w_{1}, x\right)$. We can split this integral term as

$$
I_{\xi}\left(w_{1}, x\right)=I_{\xi}\left[B_{\varrho(x)}\right]\left(w_{1}, x\right)+I_{\xi}\left[B_{\varrho(x)}^{c}\right]\left(w_{1}, x\right) .
$$

Note that for each $z \in B_{\varrho(x)}$ we have

$$
\begin{aligned}
w_{1}(x+z)-w_{1}(x) & =\left\langle D w_{1}(x+t z), z\right\rangle, \\
w_{1}(x+z)-w_{1}(x)-\left\langle D w_{1}(x), z\right\rangle & =\frac{1}{2}\left\langle D^{2} w_{1}(x+s z) z, z\right\rangle,
\end{aligned}
$$

for some $s, t \in(0,1)$. We recall that the first equality is used in the integral defining $I_{\xi}\left[B_{\varrho(x)}\right]\left(w_{1}, x\right)$ when $\sigma<1$, and the second is used in the case $\sigma \geq 1$. Now, direct computations on the derivatives of $w_{1}$ drives us to

$$
\begin{aligned}
\left\langle D^{2} w_{1}(x+s z) z, z\right\rangle & \leq C_{1} \gamma d_{0}(x)^{\gamma-2}|z|^{2} \\
\left\langle D w_{1}(x+t z), z\right\rangle & \leq C_{1} \gamma d_{0}(x)^{\gamma-1}|z| .
\end{aligned}
$$

for all $z \in B_{\varrho(x)}, s, t \in(0,1)$. Thus, using these inequalities on the corresponding form of $I_{\xi}\left[B_{\varrho(x)}\right]\left(w_{1}, x\right)$, using that $\varrho(x) \leq d_{0}(x)$ and applying (M2), we arrive at

$$
I_{\xi}\left[B_{\varrho(x)}\right]\left(w_{1}, x\right) \leq C_{R} C_{1} \varrho(x)^{\gamma-\sigma} .
$$


Concerning the estimate of $I_{\xi}\left[B_{\varrho(x)}^{c}\right]\left(w_{1}, x\right)$, we write

$$
I_{\xi}\left[B_{\varrho(x)}^{c}\right]\left(w_{1}, x\right) \leq \int_{B_{\varrho(x)}^{c}}\left[w_{1}(x+z)-w_{1}(x)\right] \nu_{\xi}(d z)+\left|D w_{1}(x)\right| \int_{B \backslash B_{\varrho(x)}}|z| \nu_{\xi}(d z),
$$

and we suppress the last integral term in the case $\sigma<1$. Using the definition of $w_{1}$ we get from the above inequality that

$$
\begin{aligned}
I_{\xi}\left[B_{\varrho(x)}^{c}\right]\left(w_{1}, x\right) \leq & \int_{B \backslash B_{\varrho(x)}}\left[w_{1}(x+z)-w_{1}(x)\right] \nu_{\xi}(d z)+C_{1} r^{\gamma} \int_{B^{c}} \nu_{\xi}(d z) \\
& +C_{1} \gamma d_{0}(x)^{\gamma-1} \int_{B \backslash B_{\varrho(x)}}|z| \nu_{\xi}(d z),
\end{aligned}
$$

where, as before, the last integral does not exist if $\sigma<1$. Since $w_{1}$ is $\gamma$-Hölder continuous we have $w_{1}(x+z)-w_{1}(x) \leq C_{1}|z|^{\gamma}$. Using this together with (M1) (see (2.1)) we can write

$$
I_{\xi}\left[B_{\varrho(x)}^{c}\right]\left(w_{1}, x\right) \leq C_{R} C_{1}\left(h_{\gamma, \sigma}(\varrho(x))+r^{\gamma}+d_{0}(x)^{\gamma-1} h_{1, \sigma}(\varrho(x))\right),
$$

where the last term inside the parentheses is suppressed if $\sigma<1$. Noting that $\varrho(x) \leq d_{0}(x)<r<1$, we conclude that

$$
I_{\xi}\left[B_{\varrho(x)}^{c}\right]\left(w_{1}, x\right) \leq C_{R} C_{1} \begin{cases}h_{\gamma, \sigma}(\varrho(x))+\varrho(x)^{\gamma-1} h_{1, \sigma}(\varrho(x)), & \text { if } \sigma \geq 1 \\ h_{\gamma, \sigma}(\varrho(x)), & \text { if } \sigma<1 .\end{cases}
$$

At this point, we note that if $\sigma \geq 1$ and $\gamma \in(0,1]$, we always have $h_{\gamma, \sigma}(\varrho) \leq \varrho^{\gamma-1} h_{1, \sigma}(\varrho)$, for all $\varrho \in(0,1)$. Taking this into account we get

$$
I_{\xi}\left[B_{\varrho(x)}^{c}\right]\left(w_{1}, x\right) \leq C_{R} C_{1} \begin{cases}\varrho(x)^{\gamma-1} h_{1, \sigma}(\varrho(x)), & \text { if } \sigma \geq 1 \\ h_{\gamma, \sigma}(\varrho(x)), & \text { if } \sigma<1 .\end{cases}
$$

and joining this last inequality and (2.18) we conclude that

$$
I_{\xi}\left(w_{1}, x\right) \leq C_{R} C_{1} \begin{cases}\varrho(x)^{\gamma-1} h_{1, \sigma}(\varrho(x)), & \text { if } \sigma \geq 1 \\ h_{\gamma, \sigma}(\varrho(x)), & \text { if } \sigma<1\end{cases}
$$

2.- Estimate for $I_{\xi}\left(w_{2}, x\right)$. Analogously as the previous estimate, we write

$$
I_{\xi}\left(w_{2}, x\right)=I_{\xi}\left[B_{\varrho(x)}\right]\left(w_{2}, x\right)+I_{\xi}\left[B_{\varrho(x)}^{c}\right]\left(w_{2}, x\right) .
$$

We start with $I_{\xi}\left[B_{\varrho(x)}\right]\left(w_{2}, x\right)$. By recalling (2.15), direct computations drive us to

$$
\begin{aligned}
D w_{2}(x)= & C_{1} \gamma d_{r}^{\gamma-1}(x) D d_{0}(x), \\
D^{2} w_{2}(x)= & C_{1} \gamma d_{r}(x)^{\gamma-2} d_{0}(x)^{-1} \\
& \times\left(d_{r}(x) I_{N}+\left[(1-\gamma) d_{0}(x)-d_{r}(x)\right] D d_{0}(x) \otimes D d_{0}(x)\right),
\end{aligned}
$$

and therefore, using the above computations as a Taylor expansion of the finite difference in the integral defining $I_{\xi}\left[B_{\varrho(x)}\right]\left(w_{2}, x\right)$, we claim that

$$
I_{\xi}\left[B_{\varrho(x)}\right]\left(w_{2}, x\right) \leq C_{R} C_{1} \varrho(x)^{\gamma-\sigma} .
$$


In fact, when $\sigma<1$, using (2.2) and the above expression for $D w_{2}$, we have

$$
I_{\xi}\left[B_{\varrho(x)}\right]\left(w_{2}, x\right)=C_{1} \gamma \int_{0}^{1} \int_{B_{\varrho(x)}} d_{r}^{\gamma-1}(x+s z)\left\langle D d_{0}(x+s z), z\right\rangle \nu_{\xi}(d z) d s,
$$

but for all $s \in(0,1)$ and $z \in B_{\varrho(x)}$, we have $d_{r}(x+s z) \geq \varrho(x)$. Thus, we have

$$
I_{\xi}\left[B_{\varrho(x)}\right]\left(w_{2}, x\right) \leq C C_{1} \varrho^{\gamma-1}(x) \int_{B_{\varrho(x)}}|z| \nu_{\xi}(d z),
$$

and applying (M1) we conclude (2.20).

Now we deal with the case $\sigma \geq 1$. Since in this case

$$
I_{\xi}\left[B_{\varrho(x)}\right]\left(w_{2}, x\right)=\frac{1}{2} \int_{0}^{1} \int_{B_{\varrho(x)}}\left\langle D^{2} w_{2}(x+s z) z, z\right\rangle \nu(d z) d s,
$$

using the explicit form of $D^{2} w_{2}$ we get

$$
\begin{aligned}
& I_{\xi}\left[B_{\varrho(x)}\right]\left(w_{2}, x\right) \\
\leq & C C_{1} r \int_{0}^{1} \int_{B_{\varrho(x)}} d_{r}(x+s z)^{\gamma-2} d_{0}(x+s z)^{-1}|z|^{2} \nu_{\xi}(d z) d s,
\end{aligned}
$$

and we estimate this last integral by cases. If $d_{0}(x) \geq r / 2$ we have $\varrho(x)=$ $d_{r}(x) / 4$. Then, for $z \in B_{\varrho(x)}$ and $s \in(0,1)$ we have $3 \varrho(x) \leq d_{r}(x+s z)$ and $r / 4 \leq d_{0}(x+s z)$. Using these estimates into (2.21), we conclude

$$
I_{\xi}\left[B_{\varrho(x)}\right]\left(w_{2}, x\right) \leq C C_{1} \varrho(x)^{\gamma-2} \int_{B_{\varrho(x)}}|z|^{2} \nu_{\xi}(d z) \leq C_{R} C_{1} \varrho(x)^{\gamma-\sigma},
$$

where we have used (M2). On the other hand, if $d_{0}(x)<r / 2$ we have $\varrho(x)=d_{0}(x) / 4$. Then, for $z \in B_{\varrho(x)}$ and $s \in(0,1)$ we have $r / 4 \leq d_{r}(x+s z)$ and $3 \varrho(x) \leq d_{0}(x+s z)$. Using these estimates into (2.21), we get

$$
I_{\xi}\left[B_{\varrho(x)}\right]\left(w_{2}, x\right) \leq C C_{1} r^{\gamma-1} \varrho(x)^{-1} \int_{B_{\varrho(x)}}|z|^{2} \nu_{\xi}(d z) \leq C_{R} C_{1} \varrho(x)^{\gamma-\sigma},
$$

where we have used that $\varrho(x) \leq r$ and (M2). This concludes (2.20).

Concerning the estimate of $I_{\xi}\left[B_{\varrho(x)}^{c}\right]\left(w_{2}, x\right)$, we should be careful with the fact that $C_{2}$ may be strictly positive.

At one hand, if $C_{2}=0$, then as in the computations relative to $w_{1}$, we have

$$
w_{2}(x+z)-w_{2}(x) \leq C_{1}|z|^{\gamma} \text { for all } z \in B_{\varrho(x)}^{c},
$$

and therefore, we can write

$$
\begin{aligned}
& I_{\xi}\left[B_{\varrho(x)}^{c}\right]\left(w_{2}, x\right) \\
\leq & \int_{B_{\varrho(x)}^{c}}\left[w_{2}(x+z)-w_{2}(x)\right] \nu_{\xi}(d z)+\left|D w_{2}(x)\right| \int_{B \backslash B_{\varrho(x)}}|z| \nu_{\xi}(d z) \\
\leq & C_{1} \int_{B_{\varrho(x)}^{c}}|z|^{\gamma} \nu_{\xi}(d z)+C_{1} d_{r}(x)^{\gamma-1} \int_{B \backslash B_{\varrho(x)}}|z| \nu_{\xi}(d z),
\end{aligned}
$$


where the last integral is suppressed if $\sigma<1$. Thus, applying (M1) and using that $r<1$, we obtain from the above inequality that

$$
I_{\xi}\left[B_{\varrho(x)}^{c}\right]\left(w_{2}, x\right) \leq C_{R} C_{1}\left(h_{\gamma, \sigma}(\varrho(x))+d_{r}(x)^{\gamma-1} h_{1, \sigma}(\varrho(x))\right),
$$

where the last term does not exist if $\sigma<1$. Finally, since $\varrho(x) \leq d_{r}(x)$ we conclude

$$
I_{\xi}\left[B_{\varrho(x)}^{c}\right]\left(w_{2}, x\right) \leq C_{R} C_{1} \begin{cases}\varrho(x)^{\gamma-1} h_{1, \sigma}(\varrho(x)), & \text { if } \sigma \geq 1 \\ h_{\gamma, \sigma}(\varrho(x)), & \text { if } \sigma<1 .\end{cases}
$$

On the other hand, if $C_{2}>0$, then we have the inequality

$$
w_{2}(x+z)-w_{2}(x) \leq C_{1}+C_{2} \quad \text { for all } z \in B_{\varrho(x)}^{c} .
$$

Using this, now we can write

$$
\begin{aligned}
& I_{\xi}\left[B_{\varrho(x)}^{c}\right]\left(w_{2}, x\right) \\
\leq & \int_{B_{\varrho(x)}^{c}}\left[w_{2}(x+z)-w_{2}(x)\right] \nu_{\xi}(d z)+\left|D w_{2}(x)\right| \int_{B \backslash B_{\varrho(x)}}|z| \nu_{\xi}(d z) \\
\leq & \left(C_{1}+C_{2}\right) \int_{B_{\varrho(x)}^{c}} \nu_{\xi}(d z)+C_{1} d_{r}(x)^{\gamma-1} \int_{B \backslash B_{\varrho(x)}}|z| \nu_{\xi}(d z),
\end{aligned}
$$

where the last integral is suppressed if $\sigma<1$. Applying (M1) and using that $\varrho(x) \leq d_{r}(x)$ we conclude in this case that

$$
I_{\xi}\left[B_{\varrho(x)}^{c}\right]\left(w_{2}, x\right) \leq C_{R}\left(C_{1}+C_{2}\right) \varrho(x)^{-\sigma}+C_{R} C_{1} \varrho^{\gamma-1}(x) h_{1, \sigma}(\varrho(x)),
$$

where the last term does not exist if $\sigma<1$. Thus, since $\gamma>0$ we get

$$
I_{\xi}\left[B_{\varrho(x)}^{c}\right]\left(w_{2}, x\right) \leq C_{R}\left(C_{1}+C_{2}\right) \varrho(x)^{-\sigma} .
$$

In summary, when $C_{2}=0$, joining (2.22) and (2.20) we have

$$
I_{\xi}\left(w_{2}, x\right) \leq C_{R} C_{1} \begin{cases}\varrho(x)^{\gamma-1} h_{1, \sigma}(\varrho(x)), & \text { if } \sigma \geq 1 \\ h_{\gamma, \sigma}(\varrho(x)), & \text { if } \sigma<1,\end{cases}
$$

meanwhile, when $C_{2}>0$, using (2.23) and (2.20) we conclude that

$$
I_{\xi}\left(w_{2}, x\right) \leq C_{R}\left(C_{1}+C_{2}\right) \varrho(x)^{-\sigma} .
$$

3.- Conclusion. The estimate (2.17) comes from (2.19) and (2.24) when $C_{2}=0$, and from (2.19) and (2.25) when $C_{2}>0$. The proof is complete.

Using the last lemma we are able to prove $w$ is a strict supersolution for a problem ad-hoc to $\left(\mathrm{P}^{2}\right)$. This is established in the following two lemmas, whose main difference is whether $C_{2}$ is strictly positive or not.

Lemma 2.6. (Strict Supersolution, Case $\mathbf{C}_{\mathbf{2}}>\mathbf{0}$ ) Let $x_{0} \in \mathbb{R}^{N}, \sigma \in$ $(0,2)$ and $\left\{\nu_{x}\right\}_{x \in \mathbb{R}^{N}}$ a family of measures satisfying (M1), (M2) relative to $\sigma$. Consider $I_{x}$ as in (1.2), (2.2) associated to $\left\{\nu_{x}\right\}_{x \in \mathbb{R}^{N}}$. Let $m>\max \{1, \sigma\}$, $\theta \in[0, m)$ and $\gamma_{0}$ given in (2.5). 
Then, for each $A, b_{0}, C_{2}>0$, there exists $C_{1}>0$ large enough such that, for all $r \in(0,1)$ and $\gamma \in\left(0, \gamma_{0}\right]$, the function $w$ defined in (2.14) (relative to $x_{0}, \gamma, C_{1}, C_{2}$ and $r$ ) satisfies the inequality

$$
-\sup _{\xi \in B_{1}(x)}\left\{I_{\xi}(w, x)\right\}+b_{0}|D w(x)|^{m} \geq A \varrho(x)^{-\theta} \quad \text { for } x \in B_{r}\left(x_{0}\right) \backslash\left\{x_{0}\right\},
$$

where $\varrho$ defined in (2.16) is associated to $x_{0}$ and $r$.

Proof: Let $x \in B_{r}\left(x_{0}\right) \backslash\left\{x_{0}\right\}$. Direct computations over $w_{1}, w_{2}$ defined in (2.15) give us the expression

$$
D w(x)=C_{1} \gamma\left(d_{0}(x)^{\gamma-1}+d_{r}(x)^{\gamma-1}\right) \frac{x-x_{0}}{\left|x-x_{0}\right|},
$$

concluding that

$$
|D w(x)|=C_{1} \gamma\left(d_{0}(x)^{\gamma-1}+d_{r}(x)^{\gamma-1}\right) \geq C C_{1} \varrho(x)^{\gamma-1} .
$$

Using this together with the estimates given by Lemma 2.5 for the nonlocal term in the case $C_{2}>0$, we obtain the existence of an universal constant $\bar{C}>0$ such that for all $C_{1}, C_{2}$ and $b_{0}$, and for all $x \in B_{r}\left(x_{0}\right) \backslash\left\{x_{0}\right\}$ we have

$$
\begin{aligned}
& -\sup _{\xi \in B_{1}(x)}\left\{I_{\xi}(w, x)\right\}+b_{0}|D w(x)|^{m} \\
\geq & \bar{C}\left(b_{0} C_{1}^{m} \varrho(x)^{m(\gamma-1)}-\left(C_{1}+C_{2}\right) C_{R} \varrho(x)^{-\sigma}\right) .
\end{aligned}
$$

But since $\gamma_{0}=\min \{m-\sigma, m-\theta\} / m$ and $\gamma \leq \gamma_{0}$ we have $m(\gamma-1) \leq$ $\min \{-\sigma,-\theta\}$. Then, we conclude from (2.27) that

$$
-\sup _{\xi \in B_{1}(x)}\left\{I_{\xi}(w, x)\right\}+b_{0}|D w(x)|^{m} \geq \bar{C} \varrho(x)^{m(\gamma-1)}\left(b_{0} C_{1}^{m}-\left(C_{1}+C_{2}\right) C_{R}\right) .
$$

Hence, we arrive at (2.26) by taking

$$
C_{1}=\left(4 A\left(\bar{C} b_{0}\right)^{-1}\right)^{1 / m}+\left(4 C_{2} C_{R} b_{0}^{-1}\right)^{1 / m}+\left(2 C_{R} b_{0}^{-1}\right)^{1 /(m-1)},
$$

that is, we should take $C_{1}$ satisfying

$$
C_{1} \geq C\left(A^{1 / m}+C_{2}^{1 / m}+1\right),
$$

where $C>0$ is a constant not depending on $C_{2}$ or $A$.

Next lemma deals with the case $C_{2}=0$.

Lemma 2.7. (Strict Supersolution, Case $\mathbf{C}_{\mathbf{2}}=\mathbf{0}$ ) Let $x_{0} \in \mathbb{R}^{N}, \sigma \in$ $(0,2)$ and $\left\{\nu_{x}\right\}_{x \in \mathbb{R}^{N}}$ a family of measures satisfying (M1), (M2) relative to $\sigma$. Consider $I_{x}$ as in (1.2), (2.2) associated to $\left\{\nu_{x}\right\}_{x \in \mathbb{R}^{N}}$. Let $m>\max \{1, \sigma\}$, $\theta \in[0, m)$ and $\gamma_{0}$ defined in (2.7). Assume $C_{2}=0$.

Then, for each $A, b_{0}>0$, there exists $C_{1}>0$ large enough such that, for all $r \in(0,1)$ and $\gamma \in\left(0, \gamma_{0}\right]$, the function $w$ defined in (2.14) (relative to $x_{0}, \gamma, C_{1}$ and $r$ ) satisfies the inequality (2.26).

The proof of this lemma follows exactly as Lemma 2.6 using the estimate given by Lemma 2.5 in the case $C_{2}=0$ and the definition of $\gamma_{0}$ given in (2.7). 
Remark 2.8. As we mentioned in the introduction, the power profile of $w$ gives us the Hölder regularity for subsolutions to $\left(\overline{\mathrm{P}^{3}}\right)$. The different uses of Lemmas 2.6 and 2.7 can be described as follows: as it can be seen in the proof of Theorem 2.1 below, the application of Lemma [2.6 under a correct choice of $C_{2}>0$ allows us to localize the arguments to obtain an interior Hölder regularity with a Hölder seminorm (cast by $C_{1}$ ) which is independent of the distance to the boundary, a key fact to conclude the regularity up to the boundary. However, the discontinuity of $w$ due to $C_{2}>0$ implies a "worse" bound for $I_{x}(w)$ (see Lemma 2.5), restricting the values of the Hölder exponent if we look for regularity up to the boundary, no matter the nonlocal operator has censored nature or not.

On the other hand, Lemma 2.7 is used in the proof of Theorem 2.2, where no localization is needed. Thus, the "better" bounds for $I_{x}(w)$ given by Lemma 2.5 allows to obtain interior Hölder regularity with "more natural" exponents.

2.3. Proofs of the Main Theorems. We start with the regularity result up to the boundary.

Proof of Theorem [2.1: Applying Lemma 2.4, we see that $u$ satisfies the censored equation

$-I_{\Omega}(u, x)+\frac{b_{0}}{2}|D u|^{m} \leq A\left(d_{\Omega}(x)^{-\theta}+1\right)+C\left(\operatorname{osc}_{\Omega_{\nu}}(u)+1\right) d_{\Omega}(x)^{-\sigma}, \quad x \in \Omega$,

where $C>0$ is the constant given in Lemma2.4. If we define $\eta=\max \{\sigma, \theta\}$, in particular we see that $u$ satisfies the viscosity inequality

$$
-I_{\Omega}(u, x)+\frac{b_{0}}{2}|D u|^{m} \leq \tilde{A} d_{\Omega}(x)^{-\eta}, \quad x \in \Omega,
$$

where

$$
\tilde{A}=A\left(1+\operatorname{diam}(\Omega)^{\eta}\right)+C\left(\operatorname{osc}_{\Omega_{\nu}}(u)+1\right) .
$$

From this point, we will argue over equation (2.29).

Let $x_{0} \in \Omega$ and denote $R=\left|x_{0}\right|+1$. Consider $\gamma_{0}$ as in (2.5), and for $C_{1}, C_{2}>0$ to be fixed later and $r=\min \left\{1, d_{\Omega}\left(x_{0}\right)\right\} / 4$, consider $w$ as in (2.14) (with $\gamma=\gamma_{0}$ ) associated to these parameters.

Denote

$$
M:=\sup \left\{u(x)-u\left(x_{0}\right)-w(x): x \in \bar{\Omega}\right\} .
$$

The aim is to prove that for suitable $C_{1}>0$ we get $M \leq 0$, which implies easily the Hölder continuity of $u$. We argue by contradiction, assuming that $M>0$. Choosing

$$
C_{2} \geq \operatorname{osc}_{\Omega_{\nu}}(u)
$$

by definition of $w$, for each $x \in \bar{\Omega} \backslash \bar{B}_{r}\left(x_{0}\right)$ we have

$$
u(x)-u\left(x_{0}\right)-w(x) \leq \operatorname{osc}_{\Omega_{\nu}}(u)-\left(2 C_{1} r^{\gamma}+\operatorname{osc}_{\Omega_{\nu}}(u)\right)<0 .
$$


Hence, by the upper semicontinuity of $u-w$, it follows that the supremum defining $M$ is attained in $\bar{B}_{r}\left(x_{0}\right)$. Moreover, since $w\left(x_{0}\right)=0$, the point attaining the maximum in $M$ is in $\bar{B}_{r}\left(x_{0}\right) \backslash\left\{x_{0}\right\}$.

Let $A_{0}>0$ be fixed later. By Lemma 2.6. we can consider $C_{1}$ large enough in order to have

$$
-\sup _{\xi \in B_{1}(x)}\left\{\tilde{I}_{\xi}(w, x)\right\}+\frac{b_{0}}{2}|D w(x)|^{m} \geq A_{0} \varrho(x)^{-\eta}, \quad x \in B_{r}\left(x_{0}\right) \backslash\left\{x_{0}\right\},
$$

in fact, by (2.28) it is sufficient to take

$$
C_{1} \geq C\left(A_{0}^{1 / m}+C_{2}^{1 / m}+1\right)
$$

for some universal constant $C>0$. Doubling variables and penalizing, we consider

$$
M_{\epsilon}:=\sup \{\Phi(x, y):(x, y) \in \bar{\Omega} \times \bar{\Omega}\},
$$

where $\Phi(x, y)=u(x)-u\left(x_{0}\right)-w(y)-\epsilon^{-2}|x-y|^{2}$.

By classical arguments in the viscosity theory, we have $M_{\epsilon} \geq M>0$ for all $\epsilon>0$ and the supremum in $M_{\epsilon}$ is attained at $(\bar{x}, \bar{y}) \in \bar{\Omega} \times \bar{\Omega}$ with $\bar{y} \in \bar{B}_{r}\left(x_{0}\right) \backslash\left\{x_{0}\right\}$, which in addition satisfies the following properties

$$
\epsilon^{-2}|\bar{x}-\bar{y}|^{2} \rightarrow 0 ; \quad \bar{x}, \bar{y} \rightarrow x^{*} ; \quad u(\bar{x}) \rightarrow u\left(x^{*}\right), \quad \text { as } \epsilon \rightarrow 0,
$$

where $x^{*} \in \bar{B}_{r}\left(x_{0}\right) \backslash\left\{x_{0}\right\}$ attains the supremum defining $M$. In particular, $\bar{y} \neq x_{0}$ for all $\epsilon>0$. Moreover, note that the function

$$
-\Phi(\bar{x}, \cdot): y \mapsto w(y)-\left(u(\bar{x})-u\left(x_{0}\right)-\epsilon^{-2}|\bar{x}-y|^{2}\right)
$$

has a global minimum point at $\bar{y} \in \bar{B}_{r}\left(x_{0}\right) \backslash\left\{x_{0}\right\}$ for all $\epsilon>0$. We claim that this fact implies $\bar{y} \notin \partial B_{r}\left(x_{0}\right)$ for each $\epsilon>0$. Otherwise, denoting $\xi=\left(x_{0}-\bar{y}\right) /\left|x_{0}-\bar{y}\right|$ we have $\bar{y}+s \xi \in B_{r}\left(x_{0}\right)$ for each $0<s<r$. Therefore $-\Phi(\bar{x}, \bar{y}) \leq-\Phi(\bar{x}, \bar{y}+s \xi)$, which implies by definition of $w$ in (2.14)

$$
0 \leq s^{-1}(w(\bar{y})-w(\bar{y}+s \xi)) \leq \epsilon^{-2}(-2\langle\bar{x}-\bar{y}, \xi\rangle+s)
$$

and

$$
0 \leq C_{1}\left(s^{-1}\left(r^{\gamma}-(r-s)^{\gamma}\right)+s^{\gamma-1}\right) \leq \epsilon^{-2}(-2\langle\bar{x}-\bar{y}, \xi\rangle+s) .
$$

Making $s \rightarrow 0$ we arrive at a contradiction, concluding the claim. Hence, for all $\epsilon>0$, there exists $r_{\epsilon} \in(0, r)$ such that $r_{\epsilon}<\left|\bar{y}-x_{0}\right|<r-r_{\epsilon}$.

On the other hand, using that $(\bar{x}, \bar{y})$ is a maximum point for $\Phi$, denoting $h=x-y$ and $\bar{h}=\bar{x}-\bar{y}$ we have

$$
u(h+y)-w(y)-\epsilon^{-2}|h|^{2} \leq u(\bar{h}+\bar{y})-w(\bar{y})-\epsilon^{-2}|\bar{h}|^{2},
$$

for each $y \in \bar{\Omega}$ and $h$ such that $y+h \in \bar{\Omega}$. Hence, we conclude

$$
\bar{u}(y)-w(y) \leq \bar{u}(\bar{y})-w(\bar{y}) \quad \text { for all } y \in \Omega-\bar{h},
$$


where $\bar{u}(y):=u(\bar{h}+y)$ for each $y \in \Omega-\bar{h}$. In particular, $\bar{y}$ is a maximum point for $\bar{u}-w$ in $\Omega-\bar{h}$. Now, a simple translation argument over equation (2.29) allows us to prove that $\bar{u}$ satisfies the equation

$$
-\tilde{I}_{x+\bar{h}}(\bar{u}, x)+\frac{b_{0}}{2}|D \bar{u}(x)|^{m} \leq \tilde{A} d_{\Omega}^{-\eta}(x+\bar{h}), \quad x \in \Omega-\bar{h},
$$

in the viscosity sense. Since $|\bar{h}| \rightarrow 0$ as $\epsilon \rightarrow 0$, for all $\epsilon$ small enough we have $\bar{y} \in B_{r}\left(x_{0}\right) \subset \Omega-\bar{h}$. Recalling $w$ is smooth at $\bar{y}$ we can use it as a test function for $\bar{u}$ at $\bar{y}$, concluding the inequality

$$
-\tilde{I}_{\bar{y}+\bar{h}}(w, \bar{y})+\frac{b_{0}}{2}|D w(\bar{y})|^{m} \leq \tilde{A} d_{\Omega}^{-\eta}(\bar{y}+\bar{h})
$$

but since $\bar{y}+\bar{h} \in B_{1}(\bar{y})$ for $\epsilon$ small enough, using (2.32) we get

$$
A_{0} \varrho^{-\eta}(\bar{y}) \leq \tilde{A} d_{\Omega}^{-\eta}(\bar{y}+\bar{h}) .
$$

Note that for each $x \in B_{r}\left(x_{0}\right)$ we have $\varrho(x) \leq d_{\Omega}(x)$ and since $\eta \geq 0$, we get from the above inequality that

$$
A_{0} d_{\Omega}^{-\eta}(\bar{y}) \leq \tilde{A} d_{\Omega}^{-\eta}(\bar{y}+\bar{h}) .
$$

At this point, recalling $\bar{h} \rightarrow 0$ and $\bar{y} \rightarrow x^{*} \in \bar{B}_{r}\left(x_{0}\right)$ as $\epsilon \rightarrow 0$, taking limits in the above inequality we arrive at a contradiction previously fixing

$$
A_{0} \geq \tilde{A}+1 \text {. }
$$

Thus, for each $x_{0} \in \Omega$ and $r \leq d_{\Omega}\left(x_{0}\right) / 4$, we have

$$
|u(x)-u(y)| \leq C_{1}|x-y|^{\gamma_{0}} \quad \text { for all } x, y \in B_{r}\left(x_{0}\right),
$$

from which we conclude the local Hölder continuity. In the case the boundary is $C^{1,1}$, from the above inequality we note that for each $B_{r}\left(x_{0}\right) \subset \Omega$, the Hölder exponent and seminorm of $u$ in $B_{r}\left(x_{0}\right)$ does not depend on $r$, and applying the method used by Barles in [4] (see also [13]) we can extend the Hölder regularity up to the boundary.

Finally, we recall that by (2.35), (2.30), (2.33) and the choice of $C_{2}$ in (2.31), the constant $C_{1}$ leading to the contradiction has the form

$$
C_{1} \geq C\left(A^{1 / m}+\operatorname{osc}_{\Omega_{\nu}}(u)^{1 / m}+1\right),
$$

for some constant $C>0$ depending on the data.

A very important consequence of the previous result is the following control of the oscillation.

Corollary 2.9. (Oscillation Bound) Let $\Omega \subset \mathbb{R}^{N}$ be open and bounded with a $C^{1,1}$ boundary, and assume the hypotheses of Theorem 2.1 hold. Assume further the nonlocal operator has a censored nature, that is, the family of measures $\left\{\nu_{x}\right\}_{x \in \mathbb{R}^{N}}$ satisfies the censored condition (2.8). Then, there exists $K>0$ such that, for each bounded viscosity subsolution u of ([?]), we have

$$
\operatorname{osc}_{\Omega}(u) \leq K
$$


Proof: The choice of $C_{1}$ given by (2.36) in Theorem 2.1 leads us to

$$
|u(x)-u(y)| \leq C\left(A^{1 / m}+\operatorname{osc}_{\Omega_{\nu}}(u)^{1 / m}+1\right)|x-y|^{\gamma_{0}}, \quad \text { for all } x, y \in \bar{\Omega},
$$

where $\gamma_{0}$ is given by (2.5). Now, by (2.8) we have $\operatorname{osc}_{\Omega_{\nu}}(u)=\operatorname{osc}_{\Omega}(u)$ and by compactness of $\bar{\Omega}$, there exists $\underline{x}, \bar{x} \in \bar{\Omega}$ such that $\operatorname{osc}_{\Omega}(u)=u(\bar{x})-u(\underline{x})$. Then, we can write

$$
\operatorname{osc}_{\Omega}(u) \leq C\left(A^{1 / m}+\operatorname{osc}_{\Omega}(u)^{1 / m}+1\right),
$$

from where we obtain the result since $m>1$.

Note that for noncensored problems, we can provide global oscillation bounds as in the last corollary if we a priori know that $\operatorname{osc}_{\Omega_{\nu}}(u)=\operatorname{osc}_{\Omega}(u)$.

Proof of Theorem [2.2: Let $x_{0} \in \Omega$, denote $R=\left|x_{0}\right|+1$ and fix $r=$ $\min \left\{1, d_{\Omega}\left(x_{0}\right)\right\} / 4$. Consider $\gamma_{0}$ as in (2.7) and for $C_{1}>0$ to be fixed later, define $w$ as in (2.14) (with $\gamma=\gamma_{0}$ ) associated to these parameters.

Since the proof follows the same lines of Theorem 2.1, we will be sketchy in the current proof bringing light on its contrasts. The first difference is that this time we do not censorize the equation (since it would restrict the Hölder exponent, see Lemma 2.4).

Denote

$$
M:=\sup \left\{u(x)-u\left(x_{0}\right)-w(x): x \in \mathbb{R}^{N}\right\} .
$$

The aim is to prove that for suitable $C_{1}>0$ we get $M \leq 0$. We argue by contradiction, assuming that $M>0$. Note that choosing

$$
C_{1} r^{\gamma_{0}} \geq \operatorname{osc}_{\Omega_{\nu}}(u)
$$

and by the upper semicontinuity of $u-w$ we have the supremum defining $M$ is attained in $\bar{B}_{r}\left(x_{0}\right)$.

Let $A_{0}>0$ be fixed later. Enlarging $C_{1}$ if it is necessary, by Lemma 2.7 we can write

$$
-\sup _{\xi \in B_{1}(x)}\left\{I_{\xi}(w, x)\right\}+b_{0}|D w(x)|^{m} \geq A_{0} \varrho(x)^{-\theta}, \quad x \in B_{r}\left(x_{0}\right) \backslash\left\{x_{0}\right\} .
$$

Doubling variables and penalizing, we consider

$$
M_{\epsilon}:=\sup \left\{\Phi(x, y):(x, y) \in \mathbb{R}^{N} \times \mathbb{R}^{N}\right\},
$$

where $\Phi(x, y)=u(x)-u\left(x_{0}\right)-w(y)-\epsilon^{-2}|x-y|^{2}$. By classical arguments in the viscosity theory, we have $M_{\epsilon} \geq M>0$ for all $\epsilon>0$ and the supremum in $M_{\epsilon}$ is attained at $(\bar{x}, \bar{y})$ with $\bar{x}, \bar{y} \in \mathbb{R}^{N}$ with $\bar{y} \in \bar{B}_{r}\left(x_{0}\right) \backslash\left\{x_{0}\right\}$, which in addition satisfies (2.34) where $x^{*} \in B_{r}\left(x_{0}\right) \backslash\left\{x_{0}\right\}$ attains the supremum in (2.37).

If $\gamma_{0}<1$, then we can prove that $\bar{y} \notin \partial B_{r}\left(x_{0}\right)$ in the same way as in Theorem 2.1 using that $w$ satisfies a state constraint problem on $\partial B_{r}\left(x_{0}\right)$. If $\gamma_{0}=1$ (which is the case of $\theta=0$ and $\sigma<1$ ), then we consider $w$ with $\gamma<\gamma_{0}$ and continue with the proof, taking into account that the Hölder seminorm does not change as $\gamma \rightarrow \gamma_{0}$. 
From this point, we follow the remaining lines of Theorem 2.1, taking $A_{0}$ large in terms of $A$ arising in $\left(\underline{\mathrm{P}^{\prime}}\right)$.

2.4. Examples. In this section we provide some examples of nonlocal terms and Hamiltonians for which our results hold.

We start with the assumptions over the nonlocal term. As we mentioned before, assumptions (M1) and (M2) are intended as a restriction on the order of the operator, which is less or equal than $\sigma$. In the case of $x$-independent operators, that is the case when there exists a measure $\nu$ such that the family $\left\{\nu_{x}\right\}_{x \in \mathbb{R}^{N}}$ defining $I_{x}$ satisfies $\nu_{x}=\nu$ for each $x \in \mathbb{R}^{N}$, the operator may range from zero order operators (when $\nu$ is finite, see [16]) to the factional Laplacian of order $s$ for $s \leq \sigma$, passing through operators which are not uniformly elliptic in the sense of Caffarelli and Silvestre [12, as it is the case of measures with the form

$$
\nu(d z)=\mathbf{1}_{\mathbb{H}_{+}}(z)|z|^{-(N+s)} d z,
$$

where $0<s \leq \sigma$ and $\mathbb{H}_{+}=\left\{\left(z^{\prime}, z_{N}\right) \in \mathbb{R}^{N}: z_{N}>0\right\}$. Another interesting example of such non-uniformly elliptic operators is given by operators with "orthogonal diffusion", for example in the case $\nu$ has the form

$$
\nu(d z)=\left|z_{2}\right|^{-\left(N+s_{2}\right)} d z_{2} \otimes \delta_{0}\left(z_{1}\right) d z_{1}+\left|z_{1}\right|^{-\left(N+s_{1}\right)} d z_{1} \otimes \delta_{0}\left(z_{2}\right) d z_{2}
$$

where $z=\left(z_{1}, z_{2}\right)$ with $z_{i} \in \mathbb{R}^{d_{i}}, i=1,2$ and $N=d_{1}+d_{2}$, and $0<s_{1}, s_{2} \leq \sigma$. Here $\delta_{0}$ denotes the Dirac measure supported at 0 and $\otimes$ denotes the measure product. In this case, such a measure gives rise to an operator which is the sum of fractional Laplacians in each direction $z_{i}, i=1,2$.

Concerning $x$-dependent nonlocal operators, the classical example comes from measures $\nu_{x}$ with the form

$$
\nu_{x}(d z)=K(x, z) \nu(d z),
$$

where $\nu$ is an $x$-independent Lévy measure and $K: \mathbb{R}^{N} \times \mathbb{R}^{N} \rightarrow \mathbb{R}$ is a nonnegative function such that $K(\cdot, z) \in L_{l o c}^{\infty}\left(\mathbb{R}^{N}\right)$ for all $x \in \mathbb{R}^{N}$, and $K(x, \cdot) \in L^{\infty}\left(\mathbb{R}^{N}\right)$ for all $x \in \mathbb{R}^{N}$. As a particular case we have the weighted fractional Laplacian

$$
-I_{x}(u, x)=K(x)(-\Delta)^{\sigma} u(x),
$$

where $K$ is bounded and nonnegative.

We highlight that in view of Lemma 2.4, the regularity results apply to censored operators defined in (2.11), where we recall that the measures defining them has the form (2.9).

Concerning $H$, we note that the structure of the Hamiltonian is encoded by the inequality (2.3). Thus, given $\sigma$ and $m>\max \{1, \sigma\}$, our results apply to $H$ with the form

$$
H(x, p)=b(x)|p|^{m}+a_{1}(x)|p|^{l}+\left\langle a_{2}(x), p\right\rangle-f(x),
$$


where $x, p \in \mathbb{R}^{N}, b \geq b_{0}>0,0<l<m$ and $a_{1}, a_{2}, f$ bounded. In the case $m \leq 1$ we can consider

$$
H(x, p)=b(x)|p|^{m}+a_{1}(x)|p|^{l}-f(x),
$$

with $b, a_{1}, l$ and $f$ as above. Of course, we can replace the main power $|p|^{m}$ by $\phi(x, p)|p|^{m}$, where the function $\phi: \mathbb{R}^{N} \times \mathbb{R}^{N} \rightarrow \mathbb{R}$ satisfies $\phi \geq \phi_{0}$ for some constant $\phi_{0}>0$.

2.5. Regularity Results for the Sublinear Case. In this subsection we provide a regularity results in the case $\sigma<m \leq 1$.

Theorem 2.10. Let $\Omega \subseteq \mathbb{R}^{N}$ be a bounded domain and $\sigma \in(0,1)$. Let $I_{x}$ as in (2.2) associated to a family of measures $\left\{\nu_{x}\right\}_{x \in \mathbb{R}^{N}}$ satisfying (M1), (M2) relative to $\sigma$. Let $m \in(\sigma, 1], \theta \in[0, m)$ and $\gamma_{0}$ as in (2.5).

Then, for each $b_{0}, A>0$ and $\gamma<\gamma_{0}$, any bounded viscosity subsolution $u: \mathbb{R}^{N} \rightarrow \mathbb{R}$ to the equation $\left(\mathrm{P}^{\prime}\right)$ is locally Hölder continuous in $\Omega$ with Hölder exponent $\gamma$. If $\Omega$ has $C^{1,1}$ boundary, then $u$ is $\gamma$-Hölder continuous in $\Omega$ and can be extended as a Hölder continuous function on $\bar{\Omega}$.

The Hölder seminorm depends on the data and $\operatorname{osc}_{\Omega_{\nu}}(u)$, where $\Omega_{\nu}$ is defined in (2.4).

Proof: As in Theorem 2.1, we start with the analogous of Lemma 2.6, Let $r>0$, consider $x_{0} \in \mathbb{R}^{N}$, define $d_{0}, d_{r}$ as in (2.13) and $\varrho$ as in (2.16). Let $w$ defined in (2.14) associated to these parameters and $\gamma<\gamma_{0}$. Let $A, b_{0}>0$. Performing the same computations as in Lemma 2.6 we arive at inequality (2.27), that is

$$
\begin{aligned}
& -\sup _{\xi \in B_{1}(x)}\left\{I_{\xi}(w, x)\right\}+b_{0}|D w(x)|^{m} \\
\geq & \bar{C}\left(b_{0} C_{1}^{m} \varrho(x)^{m(\gamma-1)}-\left(C_{1}+C_{2}\right) C_{R} \varrho(x)^{-\sigma}\right),
\end{aligned}
$$

for all $x \in B_{r}\left(x_{0}\right) \backslash\left\{x_{0}\right\}$. Since this time $m(\gamma-1)<-\sigma$ and $\varrho(x) \leq r$ for each $x \in B_{r}\left(x_{0}\right)$, we can take $r=r\left(C_{1}, C_{2}, b_{0}\right)$ small such that

$-\sup _{\xi \in B_{1}(x)}\left\{I_{\xi}(w, x)\right\}+b_{0}|D w(x)|^{m} \geq \frac{\bar{C} b_{0} C_{1}^{m}}{2} \varrho(x)^{m(\gamma-1)}, \quad x \in B_{r}\left(x_{0}\right) \backslash\left\{x_{0}\right\}$.

By the choice of $\gamma<\gamma_{0}$, we see that $m(\gamma-1) \leq-\theta$, and therefore $w$ satisfies

$$
-\sup _{\xi \in B_{1}(x)}\left\{I_{\xi}(w, x)\right\}+b_{0}|D w(x)|^{m} \geq \bar{C} C_{1}^{m} \varrho(x)^{-\eta}, \quad x \in B_{r}\left(x_{0}\right) \backslash\left\{x_{0}\right\},
$$

with $\eta=\max \{\sigma, \theta\}$. From this point, we proceed exactly as in the proof of Theorem 2.1, where the last inequality plays the role of (2.32), concluding the result by taking $C_{1}$ large in terms of $A$.

Remark 2.11. Since $m \leq 1$, the parameter $r$ depends on $C_{2}$ in the proof of Theorem 2.10 and therefore we have a Hölder seminorm which does not give a control of the oscillation in the general case. 
Interior regularity results for the sublinear case in the flavour of Theorem 2.2 can be obtained in the same way as the previous theorem.

Theorem 2.12. Let $\Omega \subseteq \mathbb{R}^{N}$ be a bounded domain. Let $\sigma \in(0,1), I_{x}$ as in (2.2) associated to a family of measures $\left\{\nu_{x}\right\}_{x \in \mathbb{R}^{N}}$ satisfying (M1), (M2) relative to $\sigma$. Let $m \in(\sigma, 1], \theta \in[0, m)$ and $\gamma_{0}$ as in (2.7).

Then, for each $b_{0}, A>0$ and $\gamma<\gamma_{0}$, any bounded viscosity subsolution $u: \mathbb{R}^{N} \rightarrow \mathbb{R}$ to the equation (P') is locally Hölder with Hölder exponent $\gamma$. Moreover, for each $\delta>0$, the Hölder seminorm of $u$ in $\Omega^{\delta}$ depends on the data and $\operatorname{osc}_{\Omega_{\nu}}(u) \delta^{-\gamma}$.

2.6. Extension to Lévy-Ito Operators. We present an important extension of our regularity results over equations associated to nonlocal operators in Lévy-Ito form: for $x \in \mathbb{R}^{N}$ and a bounded function $\phi \in C^{2}\left(\bar{B}_{\delta}(x)\right)$ for some $\delta>0$, we consider $I_{x}^{j}$ defined as

$$
I_{x}^{j}(u, x)=\int_{\mathbb{R}^{N}}\left[u(x+j(x, z))-u(x)-\mathbf{1}_{B}\langle D u(x), j(x, z)\rangle\right] \nu(d z),
$$

where $\nu$ is a positive regular measure in $\mathbb{R}^{N}$. The function $j: \mathbb{R}^{N} \times \mathbb{R}^{N} \rightarrow \mathbb{R}^{N}$ should be understood as a jump function, whose basic assumption concerns the following bound for the jumps, which is uniform in $x$.

(J1) There exists $C_{j}>0$ such that, for all $x \in \mathbb{R}^{N}$

$$
|j(x, z)| \leq C_{j}|z| \text {. }
$$

We remark that given $\nu$ and $j$ as above, it is possible to define the associated $x$-dependent measure $\nu_{x}^{j}$ as the push forward of the measure $\nu$ through the function $j(x, \cdot)$. That is, $\nu_{x}^{j}$ is defined as

$$
\int_{\mathbb{R}^{N}} f(y) \nu_{x}^{j}(d y)=\int_{\mathbb{R}^{N}} f(j(x, z)) \nu(d z),
$$

for each measurable function $f$ satisfying $|f(z)| \leq C \min \left\{1,|z|^{2}\right\}$ for some $C>0$. It is important to remark that if $\nu$ satisfies (M1),(M2) and $j$ satisfies (J1), then $\left\{\nu_{x}^{j}\right\}_{x \in \mathbb{R}^{N}}$ satisfies (M1), (M2) too, where the associated constants now depend on $C_{j}$.

We also notice that in the case the family of measures $\left\{\nu_{x}^{j}\right\}_{x}$ satisfies (M1)(M2) with $\sigma \in(0,1)$, then we do not need to compensate the integrand and $I_{x}^{j}$ is defined as

$$
I_{x}^{j}(u, x)=\int_{\mathbb{R}^{N}}[u(x+j(x, z))-u(x)] \nu(d z) .
$$

For sake of shortness, from this point we mainly argue over $I_{x}^{j}$ with the form (2.43), but all the results are valid for $I_{x}^{j}$ with the form (2.45) when $\sigma \in(0,1)$.

The following result states the regularity result up to the boundary for Lévy-Ito problems. 
Theorem 2.13. Let $\Omega \subseteq \mathbb{R}^{N}$ be a bounded domain, $A, b_{0}>0, \sigma \in(0,2)$, a measure $\nu$ satisfying (M1)-(M2) relative to $\sigma$, and a jump function $j$ satisfying (J1). Let $I_{x}^{j}$ as in (2.43), (2.45) associated to $\nu$ and $j$. Let $m>$ $\max \{1, \sigma\}$ and $\theta \in[0, m)$.

Then, any bounded viscosity subsolution $u: \mathbb{R}^{N} \rightarrow \mathbb{R}$ to the problem

$$
-I_{x}^{j}(u, x)+b_{0}|D u(x)|^{m} \leq A d_{\Omega}(x)^{-\theta}, \quad x \in \Omega
$$

is locally Hölder continuous in $\Omega$ with Hölder exponent $\gamma_{0}$ given in (2.5), and Hölder seminorm depending on $\Omega$, the data and $\operatorname{osc}_{\Omega_{\nu j}}(u)$, where $\Omega_{\nu^{j}}$ is defined as in (2.4) relative to the familty of measures $\left\{\nu_{x}^{j}\right\}_{x \in \mathbb{R}^{N}}$ given by (2.44).

Moreover, if $\Omega$ has a $C^{1,1}$ boundary, then $u$ can be extended as a Hölder continuous function to $\bar{\Omega}$ with Hölder exponent $\gamma_{0}$.

Proof: This proof follows the lines of Theorem 2.1 and therefore we provide only a sketch of the proof in order to show how to treat the Lévy-Ito form.

1.- Technical lemmas in the Lévy-Ito context. Under the current assumptions, considering $x_{0} \in \mathbb{R}^{N}, C_{1}, C_{2}, r>0$ and $\gamma_{0}$ as in (2.5), $w$ defined in (2.14) (with $\gamma=\gamma_{0}$ ) satisfies the inequality

$$
\sup _{\xi \in B_{1}(x)}\left\{I_{\xi}^{j}(w, x)\right\} \leq C\left(C_{1}+C_{2}\right) \varrho^{-\sigma}(x), \quad \text { for all } x \in B_{r}\left(x_{0}\right) \backslash\left\{x_{0}\right\},
$$

where $\varrho$ is defined in (2.16) and this time the constant $C$ depends also on $C_{j}$ arising in (J1). This is accomplished replacing $\varrho$ by

$$
\tilde{\varrho}(x)=\min \left\{d_{0}(x), d_{r}(x)\right\} /\left(4 C_{j}\right),
$$

in the proof of Lemma 2.5. Once we get this estimate, taking $C_{1}>0$ as in (2.36) (with $C$ now depending on $C_{j}$ ) we conclude

$$
-\sup _{\xi \in B_{1}(x)}\left\{I_{\xi}^{j}(w, x)\right\}+b_{0}|D w(x)|^{m} \geq A \varrho^{-\theta}(x) \quad \text { for } x \in B_{r}\left(x_{0}\right) \backslash\left\{x_{0}\right\},
$$

following directly the arguments given in Lemma 2.6 .

2.- Censored Lévy-Ito operators. Let $u$ be a bounded subsolution to (2.46). Arguing as in Lemma 2.4, the Lévy-Ito analogous to inequality (2.29) reads as

$$
-I_{\Omega}^{j}(u, x)+\frac{b_{0}}{2}|D u|^{m} \leq A d_{\Omega}(x)^{-\theta}+C\left(\operatorname{osc}_{\Omega_{\nu}^{j}}(u)+1\right) d_{\Omega}^{-\sigma}(x), x \in \Omega,
$$

where $C$ depends on $C_{j}$ and the censored Lévy-Ito operator $I_{\Omega}^{j}$ is defined as

$$
I_{\Omega}^{j}(u, x)=\int_{x+j(x, z) \in \Omega}\left[u(x+j(x, z))-u(x)-1_{B}\langle D u(x), j(x, z)\rangle\right] \nu(d z) .
$$

3.- Conclusion. Once we localize the equation inside $\Omega$, we follow exactly the same lines of the proof of Theorem 2.1, The corresponding inequality (2.36) 
this time reads as

$$
C_{1} \geq C\left(A^{1 / m}+\operatorname{osc}_{\Omega_{\nu}^{j}}(u)+1\right)
$$

where $C$ depends on $C_{j}$.

The immediate consequence of this theorem is the corresponding control of the oscillation. Its proof follows the same lines of the one of Corollary 2.9 by using the above theorem.

Corollary 2.14. Let $\Omega \subset \mathbb{R}^{N}$ open and bounded, and assume the hypotheses of Theorem 2.13 hold. Assume further the nonlocal operator has a censored nature, that is, the family of measures $\left\{\nu_{x}^{j}\right\}_{x \in \mathbb{R}^{N}}$ defined in (2.44) satisfies the censored condition (2.8). Then, there exists $K>0$ such that, for each bounded viscosity solution of (2.46) we have

$$
\operatorname{Osc}_{\Omega}(u) \leq K \text {. }
$$

Following the directions given in Theorem 2.13, it is possible to provide an interior regularity result in the flavour of Theorem 2.2, as well as regularity results for sublinear Hamiltonians in the flavour of Theorems 2.10 and 2.12, both in the Lévy-Ito framework. Additionally, we can provide extensions for the mentioned results associated to Lévy-Ito operators when the domain is unbounded (see Remark 2.3). We omit the details.

\section{Well-Posedness for the Cauchy Problem in Lévy-Ito Form.}

The $x$-dependence of the nonlocal term represents a serious difficulty in the statement of the comparison principle for integro-differential equations (see [9]), and this comparison principle is a key tool in the study of the large time behavior of evolution equations. However, we are able to prove it in the interesting case of nonlocal operators in Lévy-Ito form defined in (2.43) and (2.45). It is why, from now on, we consider the Cauchy problem in Lévy-Ito form

$$
\begin{aligned}
\partial_{t} u(x, t)-I_{x}^{j}(u(\cdot, t), x)+H(x, D u(x, t)) & =0 \quad(x, t) \in Q, \\
u(\cdot, 0) & =u_{0} \quad x \in \mathbb{R}^{N},
\end{aligned}
$$

where we recall that $Q=\mathbb{R}^{N} \times(0, \infty)$.

We start with the assumptions. Over $\nu$ we require the classical assumption

(M) There exists $C_{\nu}>0$ such that

$$
\int_{\mathbb{R}^{N}} \min \left\{1,|z|^{2}\right\} \nu(d z) \leq C_{\nu} .
$$

We also require the following compatibility condition among $j$ and $\nu$. 
(J2) For each $\delta>0$, there exists $C_{\delta}>0$ such that, for each $x, y \in \mathbb{R}^{N}$ we have

$$
\begin{gathered}
\int_{B_{\delta}}|j(x, z)-j(y, z)|^{2} \nu(d z) \leq C_{\delta}|x-y|^{2}, \\
\int_{B \backslash B_{\delta}}|j(x, z)-j(y, z)| \nu(d z) \leq C_{\delta}|x-y| .
\end{gathered}
$$

Concerning the Hamiltonian we assume the following conditions.

(H1) There exists $m>1$ and moduli of continuity $\zeta_{1}, \zeta_{2}$ such that, for all $x, y, p, q \in \mathbb{R}^{N}$ we have

$$
H(y, p+q)-H(x, p) \leq \zeta_{1}(|x-y|)\left(1+|p|^{m}\right)+\zeta_{2}(|q|)|p|^{m-1} .
$$

(H2) Let $m$ be as in (H1). There exists $A, b_{0}>0$ such that for all $\mu \in(0,1)$ we have

$$
H(x, p)-\mu H\left(x, \mu^{-1} p\right) \leq(1-\mu)\left(b_{0}(1-m)|p|^{m}+A\right) .
$$

Note that a measure $\nu$ satisfying (M1)-(M2) satisfies (M).

Concerning (J1)-(J2), let us give an example. Consider

$$
j(x, z)=g(x) z \quad \text { for all } x, z \in \mathbb{R}^{N} .
$$

If $g: \mathbb{R}^{N} \rightarrow \mathbb{R}$ is bounded then (J1) holds but (J2) may fail. If, in addition, $g$ is Lipschitz continuous and the measure $|z| \nu(d z)$ is finite away the origin, then (J2) holds.

If $m>1$, assumption (H2) implies (2.3). Examples of Hamiltonians satisfying (H1) and (H2) are provided in subsection 2.4, see (2.41), (2.42).

Remark 3.1. In this section we will argue over nonlocal operators $I_{x}^{j}$ with the form (2.43) (that is, nonlocal operators of order $\sigma \geq 1$ ). However, the same arguments can be used to get the results related to $I_{x}^{j}$ with the form (2.45), replacing (M) by the condition

$$
\int_{\mathbb{R}^{N}} \min \{1,|z|\} \nu(d z) \leq C_{\nu}<+\infty .
$$

Our comparison principle reads as follows

Proposition 3.2. Let $\nu$ be a Lévy measure satisfying (M), $j$ satisfying (J1) and both satisfying (J2). Let $I_{x}^{j}$ defined as in (2.43) associated to $\nu$ and $j$. Assume $H$ satisfies (H1),(H2) and $u_{0} \in C_{b}\left(\mathbb{R}^{N}\right)$.

For each $T>0$, denote $Q_{T}=\mathbb{R}^{N} \times(0, T]$. Let $u, v \in L^{\infty}\left(\bar{Q}_{T}\right)$ for each $T>0$ be respective viscosity sub and supersolution to (3.1)-(3.2). Then, $u \leq v$ in $\bar{Q}$. 
We would like to mention that comparison principles for problem (3.1)(3.2) for the sublinear case (that is $m \leq 1$ in (H1)) are proven in [9] and for this reason we concentrate only in the superlinear case.

The following lemma states the initial condition for viscosity sub and supersolutions is satisfied in the classical sense.

Lemma 3.3. Let $I_{x}^{j}$ defined in (2.43) with $\nu$ satisfying $(M), j$ satisfying (J1) and $H$ satisfying (H1). Let $u, v$ be respectively a viscosity sub and supersolution to problem (3.1)-(3.2), satisfying local boundedness in $Q$. Then, $u(x, 0) \leq u_{0}(x) \leq v(x, 0)$ for all $x \in \mathbb{R}^{N}$.

We refer to [21] for a proof of the corresponding result in the secondorder setting. The proof for the current case can be obtained by adjusting the arguments showed in [21] to the nonlocal framework.

We prove Proposition 3.2 in a rather indirect way by using the following lemma, which will be also used to prove a version of the Strong Maximum Principle valid for our problem in Section 4.1 .

Lemma 3.4. Let $\sigma \in(0,2)$ and let $I_{x}^{j}$ defined in (2.43) with $\nu$ satisfying $(M), j$ satisfying (J1) and both satisfying (J2). Assume further that $j(\cdot, z) \in$ $C\left(\mathbb{R}^{N}\right)$ for each $z \in \mathbb{R}^{N}$. Let $H$ satisfying (H1),(H2). Let $u, v \in L^{\infty}\left(\bar{Q}_{T}\right)$ for all $T>0$ be respectively a sub and supersolution to (CP). Then, there exists $\bar{c}>0$ such that, for each $\mu \in(0,1)$, the function

$$
\omega(x, t):=\mu u(x, t)-v(x, t)
$$

satisfies, in the viscosity sense, the equation

$$
\partial_{t} \omega-I_{x}^{j}(\omega(\cdot, t), x)-\bar{c} \frac{\zeta_{2}(|D \omega|)^{m}}{(1-\mu)^{m-1}} \leq C A(1-\mu) \quad \text { in } Q,
$$

where $A>0$ appears in (H2), $\zeta_{2}$ appears in (H1), $\bar{c}=\left(m^{m} b_{0}^{m-1}\right)^{-1}$ and $C>0$ is an universal constant.

Proof: We start noting that if $u$ is a viscosity subsolution to (3.1), denoting $\bar{u}=\mu u$ we have

$$
\partial_{t} \bar{u}-I_{x}^{j}(\bar{u}, x)+\mu H\left(x, \mu^{-1} D \bar{u}\right) \leq 0 \quad \text { in } Q,
$$

in the viscosity sense.

Let $\left(x_{0}, t_{0}\right) \in Q$ and $\phi$ a smooth function such that $\omega-\phi$ has a strict maximum point at $\left(x_{0}, t_{0}\right)$. Let $\epsilon>0$. Doubling variables we consider the function

$$
\Phi(x, y, s, t):=\bar{u}(x, s)-v(y, t)-\tilde{\phi}(x, y, s, t),
$$

where $\tilde{\phi}(x, y, s, t)=\phi(y, t)+\epsilon^{-2}|x-y|^{2}+\epsilon^{-2}(s-t)^{2}$. By its upper semicontinuity, $\Phi$ attains its maximum over the set

$$
\mathcal{K}:=\bar{B}_{2 C_{j}}\left(x_{0}\right) \times \bar{B}_{2 C_{j}}\left(x_{0}\right) \times\left[0, t_{0}+1\right] \times\left[0, t_{0}+1\right]
$$


at a point $(\bar{x}, \bar{y}, \bar{s}, \bar{t})$. Moreover, classical argument in the viscosity theory allows us to get that, as $\epsilon \rightarrow 0$

$$
\begin{aligned}
& \bar{x}, \bar{y} \rightarrow x_{0} ; \quad \bar{s}, \bar{t} \rightarrow t_{0} ; \quad \epsilon^{-2}|\bar{x}-\bar{y}|^{2}, \epsilon^{-2}(\bar{s}-\bar{t})^{2} \rightarrow 0 \\
& \bar{u}(\bar{x}, \bar{s}) \rightarrow \bar{u}\left(x_{0}, t_{0}\right), v(\bar{y}, \bar{t}) \rightarrow v\left(x_{0}, t_{0}\right),
\end{aligned}
$$

concluding that for all $\epsilon$ suitably small, $\bar{s}, \bar{t} \in\left(0, t_{0}+1\right)$ and $\bar{x}, \bar{y} \in \bar{B}_{2 C_{j}}\left(x_{0}\right)$. Hence, using that $(x, s) \mapsto \Phi(x, \bar{y}, s, \bar{t})$ has a local maximum point at $(\bar{x}, \bar{s})$ and $(y, t) \mapsto \Phi(\bar{x}, y, \bar{s}, t)$ has a local minimum point at $(\bar{y}, \bar{t})$, we can subtract the viscosity inequality for $v$ at $(\bar{y}, \bar{t})$ to the viscosity inequality for $\bar{u}$ (given by (3.5)) at $(\bar{x}, \bar{s})$ to conclude, for each $\delta^{\prime}>0$, the inequality

$$
\mathcal{A}-I^{\delta^{\prime}} \leq 0
$$

where for $\delta^{\prime}>0$ we denote

$$
\begin{aligned}
I^{\delta^{\prime}}= & I_{\bar{x}}^{j}\left[B_{\delta^{\prime}}^{c}\right](\bar{u}(\cdot, \bar{s}), \bar{x}, \bar{p})-I_{\bar{y}}^{j}\left[B_{\delta^{\prime}}^{c}\right](v(\cdot, \bar{t}), \bar{y}, \bar{q}) \\
& +I_{\bar{x}}^{j}\left[B_{\delta^{\prime}}\right](\tilde{\phi}(\cdot, \bar{y}, \bar{s}, \bar{t}), \bar{x})-I_{\bar{y}}^{j}\left[B_{\delta^{\prime}}\right](-\tilde{\phi}(\bar{x}, \cdot, \bar{s}, \bar{t}), \bar{y}),
\end{aligned}
$$

with

$$
\begin{aligned}
& \bar{p}:=D_{x} \tilde{\phi}(\bar{x}, \bar{y}, \bar{s}, \bar{t})=2 \epsilon^{-2}(\bar{x}-\bar{y}), \\
& \bar{q}:=-D_{y} \tilde{\phi}(\bar{x}, \bar{y}, \bar{s}, \bar{t})=\bar{p}-D \phi(\bar{y}, \bar{t}),
\end{aligned}
$$

and

$$
\mathcal{A}=\left(\partial_{t} \tilde{\phi}-\partial_{s} \tilde{\phi}\right)(\bar{x}, \bar{y}, \bar{s}, \bar{t})+\mu H\left(\bar{x}, \mu^{-1} \bar{p}\right)-H(\bar{y}, \bar{q})
$$

We estimate each term of the inequality (3.7) to get the result. We start with $\mathcal{A}$, noting that taking $\epsilon=\epsilon(\mu)$ small enough, we have

$$
(1-\mu)(m-1) b_{0}-\zeta_{1}(|\bar{x}-\bar{y}|)>0 .
$$

Then, from (H1),(H2) we get

$$
\begin{aligned}
& \mu H\left(\bar{x}, \mu^{-1} \bar{p}\right)-H(\bar{y}, \bar{q}) \\
\geq & \mu H\left(\bar{x}, \mu^{-1} \bar{p}\right)-H(\bar{x}, \bar{p})+H(\bar{x}, \bar{p})-H(\bar{y}, \bar{q}) \\
\geq & (1-\mu)(m-1) b_{0}|\bar{p}|^{m}-A(1-\mu)-\zeta_{1}(|\bar{x}-\bar{y}|)\left(1+|\bar{p}|^{m}\right)-\zeta_{2}(|D \phi(\bar{y}, \bar{t})|)|\bar{p}|^{m-1} \\
\geq & \inf _{\theta \geq 0}\left\{\left((1-\mu)(m-1) b_{0}-\zeta_{1}(|\bar{x}-\bar{y}|)\right) \theta^{m /(m-1)}-\zeta_{2}(|D \phi(\bar{y}, \bar{t})|) \theta\right\} \\
& -A(1-\mu)-\zeta_{1}(|\bar{x}-\bar{y}|),
\end{aligned}
$$

that is, denoting $\tilde{c}=(m-1)^{m-1} / m^{m}$, we obtain

$$
\begin{aligned}
\mu H\left(\bar{x}, \mu^{-1} \bar{p}\right)-H(\bar{y}, \bar{q}) \geq & -\tilde{c} \frac{\zeta_{2}(|D \phi(\bar{y}, \bar{t})|)^{m}}{\left((1-\mu)(m-1) b_{0}-\zeta_{1}(|\bar{x}-\bar{y}|)\right)^{m-1}} \\
& -A(1-\mu)-\zeta_{1}(|\bar{x}-\bar{y}|),
\end{aligned}
$$

from which we conclude

$$
\begin{aligned}
\mathcal{A} \geq & \partial_{t} \phi(\bar{y}, \bar{t})-\tilde{c} \frac{\zeta_{2}(|D \phi(\bar{y}, \bar{t})|)^{m}}{\left((1-\mu)(m-1) b_{0}-\zeta_{1}(|\bar{x}-\bar{y}|)\right)^{m-1}} \\
& -A(1-\mu)-\zeta_{1}(|\bar{x}-\bar{y}|) .
\end{aligned}
$$


Now we address the estimate for $I^{\delta^{\prime}}$ in (3.7). Using the smoothness of $\phi$, (M) and (J1) we clearly have

$$
\begin{aligned}
& I_{\bar{x}}^{j}\left[B_{\delta^{\prime}}\right](\tilde{\phi}(\cdot, \bar{y}, \bar{s}, \bar{t}), \bar{x})-I_{\bar{y}}^{j}\left[B_{\delta^{\prime}}\right](-\tilde{\phi}(\bar{x}, \cdot, \bar{s}, \bar{t}), \bar{y}) \\
\leq & I_{\bar{y}}^{j}\left[B_{\delta^{\prime}}\right](\phi(\cdot, \bar{t}), \bar{y})+\epsilon^{-2} o_{\delta^{\prime}}(1) .
\end{aligned}
$$

On the other hand, since $(\bar{x}, \bar{y}, \bar{s}, \bar{t})$ is a maximum point for $\Phi$ in $\mathcal{K}$, and since $\bar{x}, \bar{y} \rightarrow x_{0}$ as $\epsilon \rightarrow 0$, for all $\epsilon$ small enough, by (J1) we have the inequality

$$
\begin{aligned}
& \bar{u}(\bar{x}+j(\bar{x}, z), \bar{s})-v(\bar{y}+j(\bar{y}, z), \bar{t})-(\bar{u}(\bar{x}, \bar{s})-v(\bar{y}, \bar{t})) \\
\leq & \phi(\bar{y}+j(\bar{y}, z), \bar{t})-\phi(\bar{y}, \bar{t})+\epsilon^{-2}\left(|\bar{x}-\bar{y}+j(\bar{x}, z)-j(\bar{y}, z)|^{2}-|\bar{x}-\bar{y}|^{2}\right),
\end{aligned}
$$

for each $z \in B_{1}$. Hence, for each $0<\delta^{\prime}<\delta<1$, using this inequality we conclude that

$$
\begin{aligned}
& I_{\bar{x}}^{j}\left[B_{\delta^{\prime}}^{c}\right](\bar{u}(\cdot, \bar{s}), \bar{x}, \bar{p})-I_{\bar{y}}^{j}\left[B_{\delta^{\prime}}^{c}\right](v(\cdot, \bar{t}), \bar{y}, \bar{q}) \\
\leq & J^{\delta}-\int_{B \backslash B_{\delta}}\langle\bar{p}, j(\bar{x}, z)-j(\bar{y}, z)\rangle \nu(d z) \\
& +I_{\bar{y}}^{j}\left[B_{\delta} \backslash B_{\delta^{\prime}}\right](\phi(\cdot, \bar{t}), \bar{y})+2 \epsilon^{-2} \int_{B_{\delta} \backslash B_{\delta^{\prime}}}|j(\bar{x}, z)-j(\bar{y}, z)|^{2} \nu(d z),
\end{aligned}
$$

where

$$
\begin{aligned}
J^{\delta}=\int_{B_{\delta}^{c}}[ & \bar{u}(\bar{x}+j(\bar{x}, z), \bar{s})-v(\bar{y}+j(\bar{y}, z), \bar{t})-(\bar{u}(\bar{x}, \bar{s})-v(\bar{y}, \bar{t})) \\
& \left.-\mathbf{1}_{B}\langle D \phi(\bar{y}, \bar{t}), j(\bar{y}, z)\rangle\right] \nu(d z) .
\end{aligned}
$$

Fixing $\delta>0$ and using (J2) together with (3.6), we conclude that

$$
\begin{aligned}
& I_{\bar{x}}^{j}\left[B_{\delta^{\prime}}^{c}\right](\bar{u}(\cdot, \bar{s}), \bar{x}, \bar{p})-I_{\bar{y}}^{j}\left[B_{\delta^{\prime}}^{c}\right](v(\cdot, \bar{t}), \bar{y}, \bar{q}) \\
\leq & J^{\delta}+I_{\bar{y}}^{j}\left[B_{\delta} \backslash B_{\delta^{\prime}}\right](\phi(\cdot, \bar{t}), \bar{y})+C_{\delta} o_{\epsilon}(1) .
\end{aligned}
$$

Hence, joining the last inequality and (3.9) in the definition of $I^{\delta^{\prime}}$, we conclude that for all $0<\delta^{\prime}<\delta$

$$
I^{\delta^{\prime}} \leq J^{\delta}+I_{\bar{y}}^{j}\left[B_{\delta}\right](\phi(\cdot, \bar{t}), \bar{y})+C_{\delta} o_{\epsilon}(1)+\epsilon^{-2} o_{\delta^{\prime}}(1),
$$

with $J^{\delta}$ defined in (3.10). Replacing the last inqueality and (3.8) into (3.7), we conclude that

$$
\begin{aligned}
& \partial_{t} \phi(\bar{y}, \bar{t})-I_{\bar{y}}^{j}\left[B_{\delta}\right](\phi(\cdot, \bar{t}), \bar{y})-J^{\delta}-\tilde{c} \frac{\zeta_{2}(|D \phi(\bar{y}, \bar{t})|)^{m}}{\left((1-\mu)(m-1) b_{0}-\zeta_{1}(|\bar{x}-\bar{y}|)\right)^{m-1}} \\
\leq & (1-\mu) A+C_{j, \delta} o_{\epsilon}(1)+\epsilon^{-2} o_{\delta^{\prime}}(1)+\zeta_{1}(|\bar{x}-\bar{y}|) .
\end{aligned}
$$

But by (J2), the continuity assumption over $j$, the semicontinuity and boundedness of $\bar{u}, v$ in each $\bar{Q}_{T}$, by using (3.6) we apply Fatou's Lemma 
concluding that for each $\delta>0$ fixed, we get

$$
\limsup _{\epsilon \rightarrow 0} J^{\delta} \leq I_{x_{0}}^{j}\left[B_{\delta}^{c}\right]\left(\omega\left(\cdot, t_{0}\right), x_{0}, D \phi\left(x_{0}, t_{0}\right)\right) .
$$

Hence, letting $\delta^{\prime} \rightarrow 0$ and $\epsilon \rightarrow 0$ in (3.11), and recalling (3.6) we conclude the desired viscosity inequality leading to (3.4).

We also require the following

Lemma 3.5. Let $I_{x}^{j}$ defined in (2.43) with $\nu$ satisfying $(M)$ and $j$ satisfying (J1). Let $\psi \in C_{b}^{2}\left(\mathbb{R}^{d}\right)$ satisfying $\|\psi\|_{C^{2}\left(\mathbb{R}^{d}\right)} \leq \Lambda$ for some $\Lambda>0$. For $\beta>0$, define the function

$$
\psi_{\beta}(x)=\psi\left(\beta^{2} x\right), \quad x \in \mathbb{R}^{N}
$$

Then, $\psi_{\beta}$ satisfies

$$
\left\|D \psi_{\beta}\right\|_{\infty} \leq \Lambda \beta^{2}, \quad\left\|D^{2} \psi_{\beta}\right\|_{\infty} \leq \Lambda \beta^{4}, \quad\left\|I_{x}^{j}\left(\psi_{\beta}, \cdot\right)\right\|_{\infty} \leq \Lambda o_{\beta}(1),
$$

where $o_{\beta}(1) \rightarrow 0$ as $\beta \rightarrow 0$.

Proof: The estimates concerning $D \psi_{\beta}, D^{2} \psi_{\beta}$ are direct. Concerning the estimate of the nonlocal term, for each $x \in \mathbb{R}^{d}$ we have

$I^{j}\left(\psi_{\beta}, x\right) \leq \Lambda \beta^{4} \int_{B}|j(x, z)|^{2} \nu(d z)+\Lambda \beta^{2} \int_{B_{1 / \beta} \backslash B}|j(x, z)| \nu(d z)+2 \Lambda \int_{B_{1 / \beta}^{c}} \nu(d z)$.

Hence, using (M) and (J1) in the right-hand side of the last inequality, we get

$$
I_{x}^{j}\left(\psi_{\beta}, x\right) \leq C_{j}^{2} C_{\nu} \Lambda \beta^{4}+C_{j} \Lambda \beta^{2} \int_{B_{1 / \beta} \backslash B}|z| \nu(d z)+2 \Lambda o_{\beta}(1) .
$$

Finally, using that $|z| \leq 1 / \beta$ in the integral term of the last inequality and applying $(\mathrm{M})$, we conclude the estimate for the nonlocal term.

Using the last three lemmas we are in position to prove the comparison principle for (3.1)-(3.2).

Proof of Proposition 3.2: Let $T>0$. We will argue over the finite horizon problem

$$
\left\{\begin{aligned}
\partial_{t} u-I_{x}^{j}(u, x)+H(x, D u) & =0 & & \text { in } Q_{T} \\
u(x, 0) & =u_{0}(x) & & x \in \mathbb{R}^{N},
\end{aligned}\right.
$$

from which the general result follows by the fact that $T$ is arbitrary.

We assume by contradiction that

$$
M:=\sup _{Q_{T}}\{u-v\}>0
$$

Denote $R=2\left(\|u\|_{L^{\infty}\left(\bar{Q}_{T}\right)}+\|v\|_{L^{\infty}\left(\bar{Q}_{T}\right)}\right)$ and consider $\psi \in C_{b}^{2}\left(\mathbb{R}^{N}\right)$ a nonnegative function with $\psi=0$ in $B, R \leq \psi \leq 2 R$ in $B_{2}^{c}$ and satisfying $\|D \psi\|_{\infty},\left\|D^{2} \psi\right\|_{\infty} \leq \Lambda$ for some $\Lambda>0$. For this function $\psi$ and $\beta>0$, consider $\psi_{\beta}$ as in (3.12). 
Now, for $\eta, \mu \in(0,1)$, consider the function

$$
\bar{\omega}(x, t)=\mu u(x, t)-v(x, t)-\eta t, \quad(x, t) \in Q .
$$

Noting that $\bar{\omega}-\psi_{\beta} \rightarrow u-v$ locally uniform in $\bar{Q}_{T}$ as $\eta, \beta \rightarrow 0$ and $\mu \rightarrow 1$, by (3.13) we see that $\bar{\omega}-\psi_{\beta}$ is strictly positive at some point in $\bar{Q}_{T}$ for all $\eta, \beta$ close to 0 and $\mu$ close to 1 . Hence, by construction of $\psi_{\beta}, \bar{\omega}-\psi_{\beta}$ attains its maximum in $\bar{Q}_{T}$ at some point $\left(x^{*}, t^{*}\right)$, and by Lemma 3.3. taking $\eta, \beta$ smaller and $\mu$ larger if it is necessary, we have $t^{*}>0$ for all such as parameters. At this point, we fix $\eta>0$ satisfying the above facts.

Now, by Lemma 3.4, $\bar{\omega}$ is a viscosity subsolution of

$$
\partial_{t} \bar{\omega}-I_{x}^{j}(\bar{\omega}(\cdot, t), x)-\bar{c} \frac{\zeta_{2}(|D \bar{\omega}|)^{m}}{(1-\mu)^{m-1}} \leq C A(1-\mu)-\eta \quad \text { in } Q_{T},
$$

and therefore we can use $\psi_{\beta}$ as a test function for $\bar{\omega}$ at $\left(x^{*}, t^{*}\right)$, concluding that

$$
-I_{x}^{j}\left(\psi_{\beta}, x^{*}\right)-\bar{c} \frac{\zeta_{2}\left(\left|D \psi_{\beta}\left(x^{*}\right)\right|\right)^{m}}{(1-\mu)^{m-1}} \leq C A(1-\mu)-\eta .
$$

Using Lemma 3.5, we conclude from the above inequality that

$$
-\left(1+\bar{c}(1-\mu)^{1-m}\right) o_{\beta}(1) \leq C A(1-\mu)-\eta .
$$

Letting $\beta \rightarrow 0$ and then $\mu \rightarrow 1$, we get the contradiction with the fact that $\eta>0$.

As it is classical in the viscosity solution's theory, Proposition 3.2 allows the application of Perron's method to conclude the existence. In this task, we introduce the additional asumption

(H0) There exists a constant $H_{0}>0$ such that $\|H(\cdot, 0)\|_{\infty} \leq H_{0}$.

This assumption allows us to build sub and supersolutions for (3.1). The existence result is the following

Corollary 3.6. Let $I_{x}^{j}$ defined as in (2.43), with $\nu$ satisfying $(M), j$ satisfying (J1) and both satisfying (J2). Assume $H \in C\left(\mathbb{R}^{N} \times \mathbb{R}^{N}\right)$ satisfies (H0)-(H2). Let $u_{0} \in C_{b}\left(\mathbb{R}^{N}\right)$. Then, there exists a unique viscosity solution $u \in C(\bar{Q}) \cap L^{\infty}\left(\bar{Q}_{T}\right)$ for all $T>0$ to problem (3.1) $-(3.2)$.

A priori bounds for the solution given in Corollary 3.6 can be derived from the application of comparison principle. Using ad-hoc sub and supersolutions, if $u$ is the solution of (3.1)-(3.2), then

$$
\|u(\cdot, t)\|_{L^{\infty}\left(\mathbb{R}^{N}\right)} \leq H_{0} t+\left\|u_{0}\right\|_{\infty}
$$

which means that for fixed time $t$, the function $x \mapsto u(x, t)$ is globally bounded in $\mathbb{R}^{N}$.

Similar results can be given for the stationary problem $(\mathbb{P})$ in the Lévy-Ito setting, namely equations with the form

$$
\lambda u-I_{x}^{j}(u, x)+H(x, D u)=0 \quad \text { in } \mathbb{R}^{N} .
$$


Proposition 3.7. Let $\lambda>0, I_{x}^{j}$ defined in (2.43) with $\nu$ satisfying $(M), j$ satisfying (J1) and both satisfying (J2). Assume H satisfies (H0)-(H2). Let $u, v$ be bounded viscosity sub and supersolution to equation (3.15). Then, $u \leq v$ in $\mathbb{R}^{N}$.

Moreover, if in addition we assume (HO), then there exists a unique viscosity solution $u \in C_{b}\left(\mathbb{R}^{N}\right)$ to equation (3.15), which satisfies

$$
\|u\|_{\infty} \leq \lambda^{-1} H_{0}
$$

\section{Application to Periodic Equations: Large Time Behavior.}

In this section we provide the large time behavior result for the problem (3.1)-(3.2) in the case the data are $\mathbb{Z}^{N}$-periodic. Hence, we will argue over the problem

$$
\begin{aligned}
\partial_{t} u-I_{x}^{j}(u(\cdot, t), x)+H(x, D u) & =0 & & \text { in } \mathcal{Q}:=\mathbb{T}^{N} \times(0,+\infty), \\
u(\cdot, 0) & =u_{0} & & \text { in } \mathbb{T}^{N}
\end{aligned}
$$

where $I_{x}^{j}$ is a nonlocal operator in Lévy-Ito form defined in (2.43) (replacing $\mathbb{R}^{N}$ by $\mathbb{T}^{N}$ ). Of course, the results obtained in this section can be readily extended to the case the Lévy-Ito operator has the form (2.45), provided the measure $\nu$ is such that $I_{x}^{j}$ has order strictly less than 1 (see Remark 3.1).

Since problem (4.1)-(4.2) is a particular case of (3.1)-(3.2), comparison principle, existence and uniqueness hold for this problem under the conditions on the data given in the statement of Proposition 3.2. In particular, for the solution $u$ of (4.1)-(4.2) we have the a priori estimate (3.14).

4.1. Strong Maximum Principle. We need some notation for the statement of the Strong Maximum Principle: let $\nu, j$ in the definition of $I_{x}^{j}$ and for $x \in \mathbb{R}^{N}$ we define inductively

$$
X_{0}(x)=\{x\}, \quad X_{n+1}(x)=\bigcup_{\xi \in X_{n}(x)}\{\xi+j(\xi, \operatorname{supp}\{\nu\})\}, \quad \text { for } n \in \mathbb{N},
$$

and

$$
\mathcal{X}(x)=\overline{\bigcup_{n \in \mathbb{N}} X_{n}} .
$$

The Strong Maximum Principle presented here relies in the nonlocallicity of the operator under the "iterative covering property"

$$
\mathcal{X}(x)=\mathbb{T}^{N}, \quad \text { for all } x \in \mathbb{T}^{N} .
$$

We can provide three interesting examples where this condition clearly holds. Of course, (4.4) depends on both $\nu$ and $j$, but we mainly focus on the structure of $\nu$ for which this condition is valid, and therefore we assume in the following examples that $j(x, z)=z$ for all $x, z \in \mathbb{R}^{N}$. In this context, the most basic example is the case where there exists $r>0$ such that

$$
B_{r} \subset \operatorname{supp}\{\nu\} .
$$


A second example where the previous property does not hold, but (4.4) remains valid, is when $\nu$ has the form (2.40), namely

$$
\nu(d z)=\left|z_{2}\right|^{-(N+\sigma)} d z_{2} \otimes \delta_{0}\left(z_{1}\right) d z_{1}+\left|z_{1}\right|^{-(N+\sigma)} d z_{1} \otimes \delta_{0}\left(z_{2}\right) d z_{2},
$$

where $\delta_{0}$ is the Dirac measure supported at 0 and $\otimes$ is the measure product.

The third example strongly takes into account the topology of the torus. In (say) $\mathbb{T}^{2}$, consider $L \subset \mathbb{T}^{2}$ a line of irrational slope, that is, $L: z_{2}=\alpha z_{1}$, with $\alpha$ irrational. Let $\tilde{\nu}$ be the 1-dimensional Haussdorff measure in $\mathbb{T}^{2}$ and let $l \subset L$ with $\tilde{\nu}(l)>0$. Then, the measure $\nu=\mathbf{1}_{l}(z) \tilde{\nu}(d z)$ satisfies the assumption (4.4).

The strong maximum principle is stated through the following

Proposition 4.1. Let $\sigma \in(0,2)$ and let $I_{x}^{j}$ defined in (2.43) with $\nu$ satisfying (M), $j$ satisfying (J1) with $j(\cdot, z) \in C\left(\mathbb{T}^{N}\right)$ for each $z \in \mathbb{R}^{N}$, and $\nu, j$ satisfying (J2) and (4.4). Consider $H$ satisfying (HO)-(H2), with $\zeta_{2}$ in (H1) such that $\zeta_{2}(s)=c|s|$ for some $c>0$. Let u be a $\mathbb{Z}^{N}$-periodic viscosity subsolution to (4.1), and $v$ a $\mathbb{Z}^{N}$-periodic viscosity supersolution to (4.1), such that there exists $\left(x_{0}, t_{0}\right) \in \mathcal{Q}$ satisfying

$$
(u-v)\left(x_{0}, t_{0}\right)=\sup _{\mathcal{Q}}\{u-v\} .
$$

Then, the function $u-v$ is constant in $\mathbb{T}^{n} \times\left[0, t_{0}\right]$. Moreover, we have

$$
(u-v)(x, t)=\sup _{x \in \mathbb{T}^{N}}\{u(x, 0)-v(x, 0)\}, \quad \text { for all }(x, t) \in \overline{\mathcal{Q}} .
$$

The following lemma is a consequence of the comparison principle, see [10].

Lemma 4.2. Assume assumptions of Proposition 3.2 hold. Let $u, v$ be locally bounded sub and supersolution to equation (4.1) and for $t \in[0,+\infty)$, define

$$
\kappa(t)=\sup _{x \in \mathbb{T}^{N}}\{u(x, t)-v(x, t)\} .
$$

Then, for all $0 \leq s \leq t$, we have $\kappa(t) \leq \kappa(s)$.

Now we are in position to prove the strong maximum principle.

Proof of Propostion 4.1: We divide the proof in several parts.

1.- Preliminaries. Under the definition of $\kappa$ in (4.5), we must prove that for each $(x, t) \in \mathbb{T}^{N} \times\left[0, t_{0}\right]$

$$
(u-v)(x, t)=\kappa(0) .
$$

However, since $\kappa\left(t_{0}\right)$ is a global maximum value of $\kappa$ in $[0,+\infty)$, by Lemma 4.2 we have $\kappa(t)=\kappa(0)$ for all $t \in\left[0, t_{0}\right]$. Hence, it is sufficient to prove that for each $\tau \in\left(0, t_{0}\right)$ we have

$$
u(x, \tau)-v(x, \tau)=\kappa(\tau), \quad \text { for all } x \in \mathbb{T}^{N},
$$

which implies the result up to $\tau=0$ and $\tau=t_{0}$ by upper-semicontinuity. 
We fix $\tau \in\left(0, t_{0}\right)$ and define the set

$$
\mathcal{M}_{\tau}=\left\{x \in \mathbb{T}^{N}:(u-v)(x, \tau)=\kappa(\tau)\right\},
$$

which is nonempty by upper-semicontinuity of $u-v$. Hence, with the above facts the proof follows by proving that $\mathcal{M}_{\tau}=\mathbb{T}^{N}$.

2.- Localization on time $\tau$. For $\eta>0$ we consider the function

$$
(x, t) \mapsto \tilde{W}(x, t):=u(x, t)-v(x, t)-\eta(t-\tau)^{2} .
$$

Note that for each $(x, t) \in \mathcal{Q}$, we have

$$
\tilde{W}(x, t) \leq \kappa(t)-\eta(t-\tau)^{2} \leq \kappa(\tau)=(u-v)\left(x_{1}, \tau\right)=\tilde{W}\left(x_{1}, \tau\right),
$$

for some $x_{1} \in \mathcal{M}_{\tau}$, and therefore the supremum of $\tilde{W}$ in $\mathcal{Q}$ is achieved, and each such as maximum point has the form $(x, \tau)$ for some $x \in \mathcal{M}_{\tau}$. Hence, we clearly have

$$
\kappa(\tau)=\sup _{(x, t) \in \mathcal{Q}} \tilde{W}(x, t)
$$

3.- Localization around a point in $\mathcal{M}_{\tau}$. From this point we fix $x_{\tau} \in \mathcal{M}_{\tau}$ and introduce a function $\psi \in C_{b}^{2}(\mathbb{R})$ with $\psi(0)=0, \psi>0$ in $\mathbb{R} \backslash\{0\}$ and $\psi(x)=4 R$ if $|x| \geq 1$, with

$$
R=\|u\|_{L^{\infty}\left(\mathbb{T}^{N} \times\left[0, t_{0}+1\right]\right)} .
$$

For $\epsilon>0, x \in \mathbb{T}^{N}$ define $\psi_{\epsilon}(x)=\psi\left(\left|x-x_{\tau}\right| / \epsilon\right)$. We remark that $\psi_{\epsilon} \epsilon$ $C_{b}^{2}\left(\mathbb{T}^{N}\right), \psi_{\epsilon}\left(x_{\tau}\right)=0, \psi_{\epsilon}>0$ in $\mathbb{R}^{N} \backslash\left\{x_{\tau}\right\}$ and for each $\epsilon>0$ its first and second derivatives are bounded, depending on $\epsilon$.

We take $0<\mu<1$, denote $\bar{u}=\mu u$ and $\omega_{\mu}=\bar{u}-v$ as in Lemma 3.4, and consider the function

$$
(x, t) \mapsto W_{\mu}(x, t):=\omega_{\mu}(x, t)-\eta|t-\tau|^{2}-(1-\mu) \psi_{\epsilon}(x) .
$$

By upper-semicontinuity of $W_{\mu}$, there exists $\left(x_{\mu}, t_{\mu}\right) \in \mathbb{T}^{N} \times\left[0, t_{0}+1\right]$ such that

$$
W_{\mu}\left(x_{\mu}, t_{\mu}\right)=\sup _{\mathbb{T}^{N} \times\left[0, t_{0}+1\right]} W_{\mu}
$$

and since $W_{\mu} \rightarrow \tilde{W}$ locally uniform on $\overline{\mathcal{Q}}$ as $\mu \rightarrow 1$ we have, up to subsequences, $\left(x_{\mu}, t_{\mu}\right) \rightarrow\left(x^{*}, \tau\right)$ as $\mu \rightarrow 1$, where $x^{*}=x^{*}(\epsilon) \in \mathcal{M}_{\tau}$.

In fact, since $\left(x_{\mu}, t_{\mu}\right)$ is maximum for $W_{\mu}$, for all $(x, t) \in \mathbb{T}^{N} \times\left[0, t_{0}+1\right]$ we have

$$
\begin{aligned}
W_{\mu}\left(x_{\mu}, t_{\mu}\right) & =(u-v)\left(x_{\mu}, t_{\mu}\right)+(\mu-1)\left(u+\psi_{\epsilon}\right)\left(x_{\mu}, t_{\mu}\right)-\eta\left(t_{\mu}-\tau\right)^{2} \\
& \geq(u-v)(x, t)+(\mu-1)\left(u+\psi_{\epsilon}\right)(x, t)-\eta(t-\tau)^{2} .
\end{aligned}
$$

In particular, taking the point $(x, t)=\left(x_{\tau}, \tau\right)$ in the right-hand side we obtain

$$
(u-v)\left(x_{\mu}, t_{\mu}\right)+(\mu-1)\left(u+\psi_{\epsilon}\right)\left(x_{\mu}, t_{\mu}\right) \geq \kappa(\tau)+(\mu-1) u\left(x_{\tau}, \tau\right) .
$$

Now, since $t_{\mu} \in\left[0, t_{0}+1\right]$ for all $\mu$ close to 1 , we have

$$
(u-v)\left(x_{\mu}, t_{\mu}\right) \leq \kappa\left(t_{\mu}\right) \leq \kappa(\tau),
$$


and replacing this into (4.6) we get

$$
u\left(x_{\mu}, t_{\mu}\right)+\psi\left(\left|x_{\mu}-x_{\tau}\right| / \epsilon\right) \leq u\left(x_{\tau}, \tau\right),
$$

that is $\psi\left(\left|x_{\mu}-x_{\tau}\right| / \epsilon\right) \leq 2 R$. By the choice of $\psi$ we conclude that $x_{\mu} \in B_{\epsilon}\left(x_{\tau}\right)$ for all $\mu$ close to 1 . Since $x_{\mu} \rightarrow x^{*} \in \mathcal{M}_{\tau}$, we conclude $x^{*} \in \bar{B}_{\epsilon}\left(x_{\tau}\right)$.

4.- Using the viscosity inequality for $\omega_{\mu}$. From the above facts, we see that the function $(x, t) \mapsto \phi(x, t):=(1-\mu) \psi_{\epsilon}(x)+\eta(t-\tau)^{2}$ is a test function for $\omega_{\mu}$ at $\left(x_{\mu}, t_{\mu}\right)$. Then, by Lemma 3.4, for each $\delta, \epsilon>0$ we have

$$
\begin{array}{r}
2 \eta\left(t_{\mu}-\tau\right)-I_{x_{\mu}}^{j}\left[B_{\delta}^{c}\right]\left(\omega_{\mu}\left(\cdot, t_{\mu}\right), x_{\mu}\right)-I_{x_{\mu}}^{j}\left[B_{\delta}\right]\left((1-\mu) \psi_{\epsilon}, x_{\mu}\right) \\
-\bar{c}(1-\mu)\left|D \psi_{\epsilon}\left(x_{\mu}\right)\right|^{m} \leq C A(1-\mu),
\end{array}
$$

but by $(\mathrm{M})$ and $(\mathrm{J} 1)$ we have

$$
I_{x_{\mu}}^{j}\left[B_{\delta}\right]\left(\psi_{\epsilon}, x_{\mu}\right) \leq C_{j}\left|D^{2} \psi_{\epsilon}\right|_{\infty} .
$$

From this, it follows that

$$
\begin{gathered}
2 \eta\left(t_{\mu}-\tau\right)-I_{x_{\mu}}^{j}\left[B_{\delta}^{c}\right]\left(\omega_{\mu}\left(\cdot, t_{\mu}\right), x_{\mu}\right) \\
-(1-\mu)\left(C_{j}\left|D^{2} \psi_{\epsilon}\right|_{\infty}+\bar{c}\left|D \psi_{\epsilon}\left(x_{\mu}\right)\right|^{m}+C A\right) \leq 0 .
\end{gathered}
$$

Note that for all $\epsilon>0$, by the smoothness of $\psi_{\epsilon}$ the term in parenthesis in (4.7) remains bounded as $\mu \rightarrow 1$, meanwhile $t_{\mu} \rightarrow \tau$. On the other hand, by the continuity of $j$ and $(\mathrm{M})$, by Dominated Convergence Theorem we get

$$
I_{x_{\mu}}^{j}\left[B_{\delta}^{c}\right]\left(\omega_{\mu}\left(\cdot, t_{\mu}\right), x_{\mu}\right) \rightarrow I_{x^{*}}^{j}\left[B_{\delta}^{c}\right]\left((u-v)(\cdot, \tau), x^{*}\right) \quad \text { as } \mu \rightarrow 1,
$$

where $x^{*} \in \mathcal{M}_{\tau}$ is such that $x^{*} \in \bar{B}_{\epsilon}\left(x_{\tau}\right)$. Recalling that $(u-v)\left(x^{*}, \tau\right)=$ $\kappa(\tau)$, letting $\mu \rightarrow 1$ in (4.7) we arrive at

$$
\int_{B_{\delta}^{c}}\left[(u-v)\left(x^{*}+j\left(x^{*}, z\right), \tau\right)-\kappa(\tau)\right] \nu(d z)=0,
$$

and since $x^{*} \in \bar{B}_{\epsilon}\left(x_{\tau}\right)$, letting $\epsilon \rightarrow 0$ we finally conclude

$$
\int_{B_{\delta}^{c}}\left[(u-v)\left(x_{\tau}+j\left(x_{\tau}, z\right), \tau\right)-\kappa(\tau)\right] \nu(d z)=0 .
$$

5.- Conclusion. Since $\delta>0$ is arbitrary, we conclude $(u-v)(x, \tau)=\kappa(\tau)$ for all $x \in X_{1}\left(x_{\tau}\right)$. Hence, we can proceed in the same way as above, concluding by induction that $(u-v)(x, \tau)=\kappa(\tau)$ for all $x \in \bigcup_{n \in \mathbb{N}} X_{n}\left(x_{\tau}\right)$. Finally, by upper-semicontinuity of $u-v$ and (4.4) we conclude the result.

Remark 4.3. In Proposition 4.1, the assumption on the continuity of $j$ can be dropped. For instance, it is used to pass to the limit in (4.8). In this direction, note that if $g \in C\left(\mathbb{T}^{N}\right)$ we can write

$$
\left|g\left(x^{*}+j\left(x^{*}, z\right)\right)-g\left(x_{\tau}+j\left(x_{\tau}, z\right)\right)\right| \leq \zeta\left(x^{*}+j\left(x^{*}, z\right)-x_{\tau}-j\left(x_{\tau}, z\right)\right),
$$


where $\zeta$ is the modulus of continuity of $g$. However, it is known that a modulus of continuity may be assumed to satisfy that $\zeta(t) \leq \zeta(\rho)+\rho^{-1} t$ for each $t, \rho>0$ (see [26]). Using this, we conclude

$\left|g\left(x^{*}+j\left(x^{*}, z\right)\right)-g\left(x_{\tau}+j\left(x_{\tau}, z\right)\right)\right| \leq \zeta(\rho)+\rho^{-1}\left(\left|x^{*}-x_{1}\right|-\left|j\left(x^{*}, z\right)-j\left(x_{\tau}, z\right)\right|\right)$

for all $\rho>0$. Hence, using (J1) we can make $x^{*} \rightarrow x_{\tau}$ and then letting $\rho \rightarrow 0$ to get the desired convergence without asking continuity on $j$.

Additionally, instead of assuming $\zeta_{2}(s)=c|s|$, it is enough to ask that

$$
\zeta_{2}(s) s^{(1-m) / m} \rightarrow 0 \quad \text { as } s \rightarrow 0 .
$$

4.2. The Ergodic Problem. Roughly speaking, solving the ergodic problem means pass to the limit as $\lambda \rightarrow 0$ in the stationary periodic problem

$$
\lambda u-I_{x}^{j}(u, x)+H(x, D u)=0 \quad x \in \mathbb{T}^{N},
$$

whose existence and uniqueness for $\lambda>0$ holds by Proposition 3.7. Hence, the required compactness of the family of solutions $\left\{u_{\lambda}\right\}$ is typically obtained by regularity results which are independent of $\lambda$.

Proposition 4.4. Let $\sigma \in(0,2)$ and $I_{x}^{j}$ defined in (2.43) with $\nu$ satisfying (M1), (M2) associated to $\sigma, j$ satisfying (J1) with $j(\cdot, z) \in C\left(\mathbb{T}^{N}\right)$ for each $z \in \mathbb{R}^{N}$, and that $\nu, j$ satisfy (J2) and (4.4). Assume $H$ satisfies (HO)-(H2), with $m>\max \{1, \sigma\}$ in (H1). Then, there exists a unique constant $c \in \mathbb{R}$ for which the stationary ergodic problem

$$
-I_{x}^{j}(u, x)+H(x, D u)=-c, \quad \text { in } \mathbb{T}^{N}
$$

has a solution $w \in C^{(m-\sigma) / m}\left(\mathbb{T}^{N}\right)$. Moreover, $w$ is the unique continuous solution of (4.10), up to an additive constant.

Proof: Let $\lambda>0$ and consider the periodic stationary problem (4.9). By Proposition 3.7 we have the existence and uniqueness of a solution $u_{\lambda}$ to this problem which, by (3.16), satisfies the estimate $\left\|u_{\lambda}\right\|_{\infty} \leq \lambda^{-1} H_{0}$. Thus, by Theorem 2.13 we show that $u_{\lambda} \in C^{(m-\sigma) / m}\left(\mathbb{T}^{N}\right)$ with Hölder seminorm independent of $\lambda$ or $\left\|u_{\lambda}\right\|_{\infty}$.

Now, denote $w_{\lambda}=u_{\lambda}-u_{\lambda}(0)$ which satisfies the equation

$$
\lambda u-\mathcal{I}_{x}^{j}(u, x)+H(x, D u)=-\lambda u_{\lambda}(0), \quad \text { in } \mathbb{T}^{N} .
$$

Using Theorem 2.13 we see that the family $\left\{w_{\lambda}\right\}_{\lambda \in(0,1)}$ is uniformly bounded and that this family is equi-Hölder with exponent $(m-\sigma) / m$. Hence, by Arzela-Ascoli Theorem, there exists $w \in C^{(m-\sigma) / m}\left(\mathbb{T}^{N}\right)$ such that $w_{\lambda} \rightarrow w$ as $\lambda \rightarrow 0$, uniformly on $\mathbb{T}^{N}$. Additionally, we have the existence of a constant $c \in \mathbb{R}$ such that $\lambda u_{\lambda}(0) \rightarrow c$ as $\lambda \rightarrow 0$. By standard stability results for viscosity solutions (see [9], 1] and [20]), we have the pair $(w, c)$ found above is a (viscosity) solution to (4.10).

If $\left(w_{i}, c_{i}\right), i=1,2$ are two solutions for (4.10), then we see that $v_{i}(x, t)=$ $w_{i}(x, t)+c_{i} t, i=1,2$ are two solutions to the Cauchy problem (4.1) with initial data $w_{i}$. Hence, by comparison principle we conclude that

$$
v_{1}(x, t)-\left\|w_{1}-w_{2}\right\|_{\infty} \leq v_{2}(x, t), \quad \text { for all }(x, t) \in \mathcal{Q},
$$


and therefore, we obtain $\left(c_{1}-c_{2}\right) t \leq 2\left\|w_{1}-w_{2}\right\|_{\infty}$. Dividing by $t$ and letting $t \rightarrow+\infty$ we obtain that $c_{1} \leq c_{2}$. Exchanging the roles of $w_{1}$ and $w_{2}$, we get $c_{1}=c_{2}=c$ and therefore $c$ is unique. Moreover, for each $t \in[0,+\infty)$ we have

$$
\sup _{x \in \mathbb{T}^{N}}\left\{v_{1}(x, t)-v_{2}(x, t)\right\}=\sup _{\mathcal{Q}}\left\{v_{1}-v_{2}\right\}=\sup _{\mathbb{T}^{N}}\left\{w_{1}-w_{2}\right\}=: m,
$$

and therefore, by Proposition 4.1 we conclude that for each $x \in \mathbb{T}^{N}$

$$
w_{1}(x)=w_{2}(x)+m,
$$

concluding the proof.

4.3. Large Time Behavior. The main result of this section is the following

Theorem 4.5. Assume assumptions of Proposition 4.4 hold. Let $u$ be the unique solution to problem (4.1) -(4.2). Then, there exists a pair $(w, c)$ solution to (4.10) such that

$$
u(x, t)-c t-w(x) \rightarrow 0, \quad \text { as } t \rightarrow+\infty,
$$

uniformly in $\mathbb{T}^{N}$.

Proof: Here we follow closely the arguments given in [10, 35] in the local framework and [6] in the nonlocal one.

We assume first that $u_{0} \in C^{2}\left(\mathbb{T}^{N}\right)$. In this case, by using comparison principle it is possible to prove that $u$ is Lipschitz in $t$ (see [35]), with Lipschitz constant

$$
C^{*}=\left\|-I_{x}^{j}\left(u_{0}, \cdot\right)+H\left(\cdot, D u_{0}\right)\right\|_{L^{\infty}\left(\mathbb{T}^{N}\right)}<\infty .
$$

Now, by recalling that (H2) implies (2.3), for each $t \in(0,+\infty)$ the function $x \mapsto u(x, t)$ is a viscosity subsolution to the problem

$$
-I_{x}^{j}(u, x)+b_{0}|D u|^{m} \leq C^{*}+H_{0},
$$

with $H_{0}$ given by (H0). Using Theorem 2.13 we conclude the unique solution to $u$ of problem (4.1)-(4.2) is in $C^{\gamma_{0}, 1}(\mathcal{Q})$, with $\gamma_{0}$ defined in (2.5).

Note that $u$ and the function $(x, t) \mapsto w(x)+c t$ are solutions to (4.1). Hence, by comparison principle we have

$$
\|u(\cdot, t)-w-c t\|_{\infty} \leq\left\|u_{0}-w\right\|_{\infty}
$$

meanwhile, if we define

$$
\kappa(t)=\max _{\mathbb{T}^{N}}\{u(\cdot, t)-w-c t\},
$$

by Lemma 4.2 we see that $\kappa$ is nonincreasing. Since in addition it is bounded there exists $\bar{\kappa} \in \mathbb{R}$ such that $\kappa(t) \rightarrow \bar{\kappa}$ as $t \rightarrow+\infty$.

Now, define the function $(x, t) \mapsto v(x, t):=u(x, t)-c t$. Using (4.12) we obtain

$$
\|v(\cdot, t)\|_{\infty} \leq\|w\|_{\infty}+\left\|u_{0}-w\right\|_{\infty}, \quad \text { for each } t \geq 0
$$


and by the fact that the family $\{v(\cdot, t)\}_{t}$ is equi-Hölder (with exponent $\gamma_{0}$ ), by Arzela-Ascoli we can extract a subsequence $\left\{v\left(\cdot, t_{k}\right)\right\}_{k}$ with $t_{k} \rightarrow \infty$ as $k \rightarrow \infty$ such that

$$
v\left(\cdot, t_{k}\right) \rightarrow \bar{v}, \quad \text { uniformly in } \mathbb{T}^{N} \text { as } k \rightarrow+\infty .
$$

Define $v_{k}(x, t)=v\left(x, t+t_{k}\right)$. Recalling that $v_{k}$ is solution to

$$
\left\{\begin{aligned}
\partial_{t} v_{k}-I_{x}^{j}\left(v_{k}(\cdot, t), x\right)+H\left(x, D v_{k}\right) & =-c & & \text { in } \mathcal{Q} \\
v_{k}(x, 0) & =v\left(x, t_{k}\right) & & x \in \mathbb{T}^{N},
\end{aligned}\right.
$$

and using comparison principle we conclude $\left\{v_{k}\right\}_{k}$ satisfies the inequality

$$
\left\|v_{k}-v_{k^{\prime}}\right\|_{L^{\infty}(\mathcal{Q})} \leq\left\|v\left(\cdot, t_{k}\right)-v\left(\cdot, t_{k^{\prime}}\right)\right\|_{\infty}
$$

for all $t \geq 0$ and $k, k^{\prime} \in \mathbb{N}$. Hence, $\left\{v_{k}\right\}_{k}$ is an uniformly bounded Cauchy sequence in $C(\mathcal{Q})$ and therefore, up to a subsequence, we conclude $v_{k} \rightarrow \tilde{v}$ in $C(\mathcal{Q})$ as $k \rightarrow \infty$, where $\tilde{v}$ solves

$$
\left\{\begin{aligned}
\partial_{t} \tilde{v}-I_{x}^{j}(\tilde{v}(\cdot, t), x)+H(x, D \tilde{v}) & =-c & & \text { in } \mathcal{Q} \\
\tilde{v}(x, 0) & =\bar{v} & & x \in \mathbb{T}^{N}
\end{aligned}\right.
$$

Using the definition of $\kappa$ given in (4.13), for each $t \geq 0$ we obtain

$$
\kappa\left(t+t_{k}\right)=\max _{\mathbb{T}^{N}}\left\{v_{k}(\cdot, t)-w\right\},
$$

and since $\left\{v_{k}\right\}_{k}$ is uniformly convergent, we can pass to the limit as $k \rightarrow \infty$ concluding that

$$
\bar{\kappa}=\max _{\mathbb{T}^{N}}\{\tilde{v}(\cdot, t)-w\} \quad \text { for each } t \in[0,+\infty),
$$

and applying Proposition 4.1, for each $(x, t) \in \mathcal{Q}$ we have

$$
\tilde{v}(x, t)=w(x)+\bar{\kappa},
$$

and therefore we have $\bar{v}=w+\bar{\kappa}$ in $\mathbb{T}^{N}$. This implies that $v(x, t) \rightarrow w+\bar{\kappa}$. But by using the definition of $v$ we have

$$
\|u(\cdot, t)-c t-w-\bar{\kappa}\|_{\infty}=\|v(\cdot, t)-v-\bar{\kappa}\|_{\infty} \rightarrow 0
$$

as $t \rightarrow \infty$. Replacing $w$ by $w+\bar{\kappa}$, we conclude the result in the case the initial data is smooth.

The general result for $u_{0} \in C\left(\mathbb{T}^{N}\right)$ follows by an approximation argument using a sequence of smooth initial data $u_{0}^{\epsilon}$ satisfying $u_{0}^{\epsilon} \rightarrow u_{0}$ uniformly in $\mathbb{T}^{N}$ as $\epsilon \rightarrow 0$. We refer to 35 . for details.

Acknowledgements: G.B. and O.L. are partially supported by the ANR (Agence Nationale de la Recherche) through ANR WKBHJ (ANR-12-BS010020). S.K. is supported by Grant-in-Aid for Scientific Research (No. 23340028) of Japan Society for the Promotion of Science. E.T. was partially supported by CONICYT, Grants Capital Humano Avanzado, Cotutela en el Extranjero and Ayuda Realización Tesis Doctoral. 


\section{REFERENCES}

[1] Alvarez, O and Tourin, A. Viscosity Solutions of Nonlinear Integro-Differential Equations Annales de L'I.H.P., section C, vol.13 (1996), no. 3, 293-317.

[2] Bardi, M. and Da Lio, F. On the Strong Maximum Principle for Fully Nonlinear Degenerate Elliptic Equations Arch. Math (Basel), 73 (4), 276-285, 1999.

[3] Barles, G. Solutions de Viscosite des Equations de Hamilton-Jacobi Collection "Mathematiques et Applications" de la SIAM, no 17, Springer-Verlag (1994).

[4] Barles, G. A Short Proof of the $C^{0, \alpha}$-regularity of Viscosity Subsolutions for Superquadratic Viscous Hamilton-Jacobi Equations and Applications Nonlinear Analysis 73 (2010) 31-47.

[5] Barles, G., Chasseigne, E., Ciomaga, A. and Imbert, C. Lipschitz Regularity of Solutions for Mixed Integro-Differential Equations. J. Diff. Eq., 252 (2012), 6012-6060.

[6] Barles, G., Chasseigne, E., Ciomaga, A. and Imbert, C. Large Time Behavior of Periodic Viscosity Solutions for Uniformly Elliptic Integro-Differential Equations Preprint.

[7] G. Barles, E. Chasseigne, C. Georgelin and E.Jakobsen On Neumann type problems for nonlocal equations set in a half space. To appear in Trans. AMS.

[8] Barles, G., Chasseigne, E. and Imbert, C. On the Dirichlet Problem for Second Order Elliptic Integro-Differential Equations Indiana U. Math. Journal, 2008.

[9] Barles, G. and Imbert, C. Second-order Eliptic Integro-Differential Equations: Viscosity Solutions' Theory Revisited. IHP Anal. Non Linéare, Vol. 25 (2008) no. 3, 567-585.

[10] Barles, G. and Souganidis, P.E. Space-time Periodic Solutions and Long-Time Behavior of Solutions of Quasilinear Parabolic Equations. SIAM J. Math. Anal., 32 (2001), 1311-1323 (electronic).

[11] Bogdan, K., Burdzy, K. and Chen Z.-Q. Censored Stable Processes. Prob. Theory and Rel. Fields, Vol. 127, Issue 1 (2003), pp 89-152.

[12] Caffarelli, L. and Silvestre, L. Regularity Theory For Nonlocal Integro-Differential Equations. Comm. Pure Appl. Math, Vol. 62 (2009), no. 5, 597-638.

[13] Capuzzo-Dolcetta, I., Leoni, F. and Porretta, A. Hölder Estimates for Degenerate Elliptic Equations with Coercive Hamiltonians. Trans. Amer. Math. Soc. 362 (9) 45114536 (2010).

[14] Cardaliaguet, P. and Cannarsa, P. Hölder Estimates in Space-Time for Viscosity Solutions of Hamilton-Jacobi Equations CPAM 63(5): 590-629 (2010).

[15] Cardaliaguet, P. and Rainer Hïder Regularity for Viscosity Solutions of Fully Nonlinear, Local or Nonlocal, Hamilton-Jacobi Equations with Superquadratic Growth in the Gradient SIAM J. Control and Optimization 49(2): 555-573 (2011).

[16] Chasseigne, E. The Dirichlet problem for some nonlocal diffusion equations. Differential Integral Equations 20 (2007), no. 12, 1389???1404.

[17] Ciomaga, A. On the Strong Maximum Principle for Second Order Nonlinear Parabolic Integro-Differential Equations Advances in Diff. Equations. 17 (2012), 635-671.

[18] Coville, J. Maximum principles, sliding techniques and applications to nonlocal equations Electronic Journal of Differential Equations, Vol. 2007(2007), No. 68, pp. 1???23.

[19] Coville, J. Remarks on the Strong Maximum Principle for Nonlocal Operators Electron. J. Differential Equations, pp. No. 66, 10, 2008.

[20] Crandall, M.G., Ishii H. and Lions, P.-L. User's Guide to Viscosity Solutions of Second Order Partial Differential Equations. Bull. Amer. Math. Soc. (N.S.), Vol. 27 (1992), no. 1, 1-67.

[21] Da Lio, F. Comparison Results for Quasilinear Equations in Annular Domains and Applications. Comm. Partial Diff. Equations, 27 (1 \& 2) 283-323 (2002).

[22] Di Neza, E., Palatucci, G. and Valdinoci, E. Hitchhiker's Guide to the Fractional Sobolev Spaces Bull. Sci. Math., 136, (2012), no. 5, 521-573. 
[23] Fukushima M. , Oshima Y. and Takeda M. Dirichlet forms and symmetric Markov processes. de Grueter Studies in Mathematics 19 (1994)

[24] Guan Q.Y. The integration by part of the regional fractional Laplacian. Commun. Math. Phys. 266, 289-329 (2006)

[25] Guan Q.Y. and Ma Z.M. Reflected symmetric $\alpha$ stable process and regional fractional Laplacian. Probab. Theory Relat. Fields 134, 649-694 (2006)

[26] Ishii, H. Existence and Uniqueness of Solutions of Hamilton-Jacobi Equations. Funkcialaj Ekvacioj, Vol. 29 (1986) 167-188.

[27] Ishii, H. and Nakamura, G. A Class of Integral Equations and Approximation of p-Laplace Equations. Calc. Var. (2010), no. 37, 485-522.

[28] Ishii, H. and Lions, P.L. Viscosity Solutions of Fully Nonlinear Second-Order Elliptic Partial Differential Equations J. Differential Equations, 83(1) 26-78, 1990.

[29] Jacob N. Pseudo differential operators and Markov process. Vol III . Markov process and Applications Imperial College Press, Princeton, 2005.

[30] Kim P. Weak convergence of censored and reflected stable processes. Stochastic Process. Appl. 116 (2006), no. 12, 1792-1814.

[31] Koike, S. A Beginner's Guide to the Theory of Viscosity Solutions. Math. Soc. of Japan, 2004.

[32] Lasry, J.M. and Lions, P.L. Nonlinear elliptic Equations with Singular Boundary Conditions and Stochastic Control with State Constraints. Math. Ann. 283, 583-630 (1989).

[33] Sayah, A. Équations d'Hamilton-Jacobi du emier Ordre Avec Termes IntégroDifférentiels. I. Unicité des solutions de viscosité. Comm. Partial Differential Equations 16 (1991), 1057-1074.

[34] Sayah, A. Équations d'Hamilton-Jacobi du emier Ordre Avec Termes IntégroDifférentiels. II. Existence de solutions de viscosité. Comm. Partial Differential Equations 16 (1991), 1075-1093.

[35] Tchamba, T.T. Large Time Behavior of Solutions of Viscous Hamilton-Jacobi Equations with Superquadratic Hamiltonian Asymptot. Anal. 66 (2010) 161-186.

[36] Topp, E. Existence and Uniqueness for Integro-Differential Equations with Dominating Drift Terms. Preprint.

Guy Barles: Laboratoire de Mathématiques et Physique Théorique (UMR CNRS 7350), FÉdération Denis Poisson (FR CNRS 2964), Université François

Rabelais Tours, PARC De Grandmont, 37200 Tours, FRANCE. Guy.Barles@lmpt.univ-tours.fr

Shigeaki Koike: Mathematical Institute, Tohoku University, Aoba, Sendai 980-8578, JAPAN. koike@math.tohoku.ac.jp

OLIVIER LEY: IRMAR, INSA DE RENNES, 35708 RENNEs, FRANCE. olivier.ley@insa-rennes.fr

Erwin Topp: Departamento de Ingeniería Matemática (UMi 2807 CNRS), Universidad de Chile, Casilla 170, Correo 3, Santiago, Chile. and Laboratoire de Mathématiques et Physique ThÉorique (CNRS UMR 7350), Université François Rabelais, PARC DE Grandmont, 37200, Tours, FRANCE. etopp@dim.uchile.cl 